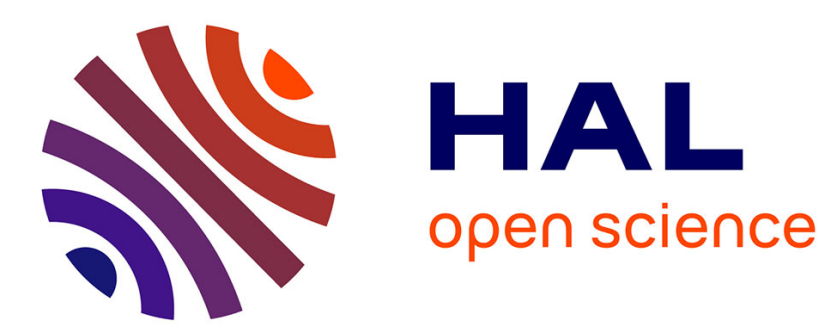

\title{
Evolution of the microstructure of unconsolidated geopolymers by thermoporometry
}

Quang Hung Nguyen, Mohamed Hanafi, Jan-philip Merkl, Jean-Baptiste d'Espinose

\section{- To cite this version:}

Quang Hung Nguyen, Mohamed Hanafi, Jan-philip Merkl, Jean-Baptiste d'Espinose. Evolution of the microstructure of unconsolidated geopolymers by thermoporometry. Journal of the American Ceramic Society, 2021, 104 (3), pp.1581-1591. 10.1111/jace.17543 . hal-03168886

\section{HAL Id: hal-03168886 https://hal.science/hal-03168886}

Submitted on 14 Mar 2021

HAL is a multi-disciplinary open access archive for the deposit and dissemination of scientific research documents, whether they are published or not. The documents may come from teaching and research institutions in France or abroad, or from public or private research centers.
L'archive ouverte pluridisciplinaire HAL, est destinée au dépôt et à la diffusion de documents scientifiques de niveau recherche, publiés ou non, émanant des établissements d'enseignement et de recherche français ou étrangers, des laboratoires publics ou privés. 


\title{
Evolution of the microstructure of unconsolidated geopolymers by
} thermoporometry

\author{
Quang Hung Nguyen*a, Mohamed Hanafi ${ }^{a}$, Jan-Philip Merkl ${ }^{b \dagger}$, Jean-Baptiste \\ d'Espinose de Lacaillerie *a
}
${ }^{a}$ Soft Matter Science and Engineering (SIMM), UMR CNRS 7615, Université PSL, ESPCI Paris, 75005, Paris, France
${ }^{b}$ BASF Construction Solutions GmbH, Dr.-Albert-Frank-Straße 32, 83308 Trostberg, Germany
* Corresponding authors e-mail:
quang-hung.nguyen@espci.fr, jean-baptiste.despinose@espci.fr

$\uparrow$ present address: Bind-X GmbH, Am Klopferspitz 19, 82152 Planegg, Germany 


\begin{abstract}
This paper studies the evolution of the pore size distribution of a fresh unconsolidated geopolymer paste between one day and a week, using thermoporometry. This was made possible by following a careful protocol for sample preparation and for analysis by differential scanning calorimetry. In contrast with nitrogen gas adsorption, this method quantifies directly the amount of water in pores. It also does not require heat and vacuum drying, thus maintaining the fragile pore structure of the unconsolidated paste. Moreover, it was found that, in a typical metakaolin-based sodium geopolymer with a 10 to $20 \mathrm{~h}$ workability period, the porosity gradually refines during the first week while the mesoporous volume is cut in half. This is probably due to the fact that the geopolymer network was still actively condensing from the activation solution. Part of the pore water never froze and, from mass balance, this residual water was attributed to the water bound in the hydration shell of the sodium counter ions. Only a minor occurrence of covalently bound protons as silanol groups was observed. The results presented here usefully complement data obtained by conventional techniques at later ages on consolidated geopolymers. It supports the growing body of literature on the structural evolution of geopolymers with time.
\end{abstract}

Keywords: Differential scanning calorimetry, pores/porosity, alkali activation, geopolymers, metakaolin 


\section{Introduction}

Geopolymers, the amorphous reaction products of silica-alumina with sodium silicate, are generally considered as a low- $\mathrm{CO}_{2}$ alternative binder. Indeed, the carbon footprints of geopolymers concretes is sometimes considered lower than that of Ordinary Portland cement (OPC) - although this is still a matter of debate[1]. Anyhow, it remains that geopolymers could advantageously replace conventional cement-based materials in specific applications such as: castable refractory, encapsulation of nuclear waste, thermal and acoustic isolation, stabilization/solidification of municipal solid waste incineration residue, and cementing of oil wells [2-6].

Contrary to high temperature glass or ceramic materials, the covalent network of geopolymers harbors a complex porous structure that remains the subject of discussions even for the simplest case of metakaolin-based geopolymers. This multiscale porosity of hardened geopolymers has attracted much attention as it controls mechanical, transport, and durability properties that are central to their foreseen usage. Most literature results concern only geopolymers pastes or mortar formulated from metakaolin (thereafter called MK geopolymers) and cannot be immediately generalized to other industrially more relevant geopolymers. They are nevertheless of high interest because MK geopolymers are synthesized from well controlled raw materials thereby forming a reproducible model material to understand the link between reactivity, pore structure and durability. Duxson et al. have studied the evolution of the mechanical properties and microstructure of sodium-based geopolymers for $\mathrm{Si} / \mathrm{Al}$ atomic ratios between 1.15 and 2.15 [7]. Samples with $\mathrm{Si} / \mathrm{Al} \geq 1.65$ appear homogeneous and have a pore range from a few micrometers down to less than $10 \mathrm{~nm}$. Several authors report an open porosity volume varying between 40 and $60 \%$ and a monomodal Brunauer-Emmett-Teller $(\mathrm{BJH})$ pore size distribution obtained by 
nitrogen adsorption of around $10 \mathrm{~nm}$ and $30 \mathrm{~nm}$ [8-10]. Recent studies have revealed that hardened MK geopolymers possess a multi-level pore network of macro- and mesopores [11-13].

How this porous structure forms during curing remains to be understood. Conventional porosimetry methods like mercury intrusion porosimetry (MIP) or nitrogen adsorption porosimetry (NAP) have established that the pore structure of MK geopolymers evolves during curing. However, this remains qualitative as these methods require not only removing water from the porous materials but also heating which in turn alters the microstructure [14-17], especially at early age when samples are mechanically fragile. Freeze-drying or vacuum-drying process allows some level of preservation of the microstructure of porous sample, however this treatment precipitates soluble species present at early ages [18]. Rovnaník studied the influence of curing temperature on the microstructure of MK geopolymer by means of mercury intrusion porosimetry (MIP) at early ages (one, three and seven days) after vacuum-drying at ambient temperature [19]. He concluded that the pore size is then situated in the mesopore region. Furthermore a slight decrease with time of the mean pore size in the range of $20 \mathrm{~nm}$ was observed. Steins et al. [20] and Benavent et al. [12] performed a very complete study of the pore structure of MK geopolymers at early age ( 3 days, 7 days) and after longer terms (up to 6 months) using NAP. In these studies, samples were freeze-dried and then degassed at $623 \mathrm{~K}\left(350{ }^{\circ} \mathrm{C}\right)$. The mean pore size appears invariant for sodium-based geopolymer whereas it slightly increases over time in case of potassium-based geopolymer. Also, the pore volume decreases over time, a phenomenon which is associated to the densification of the solid network and the partial closure of the porosity.

The foregoing literature was only concerned with geopolymers cured for at least three days when they are old enough to withstand the mechanical stress associated with MIP and NAP studies. This was justified since these studies were essentially motivated by describing usage properties 
such as durability and strength in the final material. More recently however, attempts were made to understand the formation of the pore structure before consolidation using less perturbative methods. In particular, Steins et al. gave evidence of the development of specific surface area and the progressive confinement of water during geopolymerization using SAXS and EPR probes $[20,21]$. Nevertheless, to the best of our knowledge, no studies to date have reported the evolution of the pore size distribution as such before hardening, that is, at very early ages of less than three days. Consequently, little is known about the actual mechanism leading to the microstructure despite its high relevance to workability [22]. Moreover, these circumstances impair targeting specific geopolymer pore structures via a control of the reactivity. This situation contrasts with OPC where the understanding of the link between reactivity and porous structures has allowed the design of admixtures (retarders or water reducing agents, for example) to control porosity, and ultimately to tailor low-porosity (high performance concrete) or high porosity (permeable mortars) commercial materials [23,24].

Therefore, the objective of this study is to help bridge the reactivity / porosity gap in geopolymers by providing a characterization method that effectively describes the microstructure evolution in unconsolidated MK geopolymer pastes. This study bases itself on the thermal analysis of the phase transition of water inside the porous sample: i.e. thermoporometry (TMP) based on low temperature differential scanning calorimetry (DSC) [25]. Contrary to more conventional porosimetry methods based on mercury intrusion or on nitrogen adsorption, TMP does not need to remove water by drying processes like freeze-drying, vacuum-drying or oven-drying. In this manner, it is an "in-situ" characterization method. Nevertheless, the presence of a significant quantity of alkali ions in the pore solution $[12,26]$ is a challenge to the interpretation of TMP because the underlying theory is based on the phase transition of a pure condensate (water) [27]. 
Therefore, a particular attention has been paid to the stoppage and pore solution exchange procedure. Here, a procedure derived from Chen et al [18] has been used to effectively stop the geopolymer reaction during the characterization. The results presented here thus showed that by combining stoppage, pore solution exchange and low temperature DSC (TMP), it is possible to quantify the mesoporous volume even in material with low mechanical property such as fresh geopolymer pastes. Additionally, the thermogravimetric analysis of freeze-dried geopolymers contributed to clarify the state of water in geopolymers.

\section{Experimental procedures}

\section{a. Materials}

A sodium geopolymer of composition $3.6 \mathrm{SiO}_{2} \cdot \mathrm{Al}_{2} \mathrm{O}_{3} \cdot \mathrm{Na}_{2} \mathrm{O} \cdot 12 \mathrm{H}_{2} \mathrm{O}$ was synthesized. The composition and synthesis protocol was reproduced from [21] to allow comparison with previous studies. This composition was chosen because it has relatively good mechanical properties as well as provides a long workability period (10 to 20 hours), thus allowing time to characterize the buildup of the porous network.

The chosen aluminosilicate precursor material is a metakaolin $\left(\right.$ Metamax $\left.^{\circledR}\right)$ provided by BASF. Its BET specific area and specific gravity were respectively $12 \mathrm{~m}^{2} / \mathrm{g}$ and 2.54 . The activating solution with molar composition $\mathrm{SiO}_{2} / \mathrm{Na}_{2} \mathrm{O}=1.65$ and $\mathrm{H}_{2} \mathrm{O} / \mathrm{Na}_{2} \mathrm{O}=12$ was prepared by addition of a $\mathrm{NaOH}$ solution - obtained by dissolving sodium hydroxide pellets (Sigma Aldric) in Milli-Q water (reverse osmosis purified water at $18.2 \mathrm{M} \Omega \cdot \mathrm{cm}$ ) - to a commercial sodium silicate solution (Betol ${ }^{\circledR} 52 \mathrm{~T}$, Woellner; according to the manufacturer's datasheet, its molar ratios are: $\mathrm{SiO}_{2} / \mathrm{Na}_{2} \mathrm{O}=2.1$ and $\mathrm{H}_{2} \mathrm{O} / \mathrm{Na}_{2} \mathrm{O}=12.90$ ). As $\mathrm{NaOH}$ and $\mathrm{H}_{2} \mathrm{O}$ were added to the sodium silicate solution, the equilibria between the various silicate oligomers were perturbed. To ensure that 
the solution was at equilibrium, the resulting solution was thus stirred overnight in a sealed propylene container at room temperature $\left(\sim 23{ }^{\circ} \mathrm{C}\right)$ until transparent.

The geopolymer paste was formed by mixing metakaolin into the activation solution using a Heidolph overhead stirrer (model Hei-TORQUE 200) for 5 minutes at $700 \mathrm{rpm} .62 .3 \mathrm{~g}$ Betol® 52T, $3.4 \mathrm{~g}$ sodium hydroxide, $3.7 \mathrm{~g}$ water, and $42.9 \mathrm{~g}$ of metakaolin were used for a batch of about $75 \mathrm{~mL}$. The homogeneous paste was then poured into $2 \mathrm{~mL}$ Safe-Lock tubes (Eppendorf) and cured endogenously at different times $(23 \mathrm{~h}, 48 \mathrm{~h}, 72 \mathrm{~h}$ and $168 \mathrm{~h})$. At this stage, the sample is referred to as "cured geopolymer".

The stoppage procedure combining water treatment and solvent exchange suggested by Chen et al [18] was adapted in the context of the present study. The aim of this procedure is to stop the geopolymerization reaction while avoiding precipitation of solvated species. Firstly, samples were demolded at a specific age and gently crushed into small parts $(2-3 \mathrm{~mm})$. Specimen of about $5 \mathrm{~g}$ were placed into a plastic container of $500 \mathrm{~mL}$ filled with Milli-Q water (liquid-solid ratio = 100) and rinsed in an ultrasonic bath for 30 minutes. The solid was then separated from the water by sedimentation. The pore water was exchanged with acetone by repeating the above procedure but using acetone instead of water. Acetone was again exchanged with water. For that purpose, the samples were put in a plastic container filled by Milli-Q water to ensure that the pore solution was fully replaced by water (see concentration estimations in the Supplemental materials). Water was renewed regularly, and its $\mathrm{pH}$ was monitored until it reached a neutral value in order to make sure all soluble species were fully removed (this took about two to three washing steps). Samples were then kept in water until the DSC and nitrogen adsorption experiments. These samples are called "washed geopolymers". 
In DSC experiments, excess water was removed from the surface of the sample using an adsorbent cloth in order to get the saturated-surface-dry condition. This protocol is believed to preserve the pore structure because the sample is never dried or heated all while having the added advantage of soluble species being washed out. Its effectiveness was established by analyzing the washed samples by low temperature DSC (see section II.c below) on the same day as the stoppage procedure as well as two days and more after it. The shape of the PSD remained identical and the pore volume varied only within $10 \%$, proving that the pore structure evolution was contained after stoppage (Supplemental materials, Fig. S6).

\section{b. Thermogravimetry and quantification of bound water}

In this paper, free water is defined as molecular water that can be removed from a saturated porous sample by freeze-drying while residual water is the subsequent water loss measured by thermogravimetry. The quantification of free and residual water was determined as follows. Firstly, a weighted amount of cured geopolymer was frozen by immersion in liquid nitrogen (77 $\mathrm{K}$ ) for at least 15 minutes. After freezing, the sample was placed into a round flask connected to a freeze-dryer (Christ, Alpha 1-4) and frozen pore water was removed by sublimation. The pressure and temperature in the drying chamber were kept to 0.04 mbar and $-50{ }^{\circ} \mathrm{C}$. The time of freezedrying was about 1 day for every samples. About $20 \mathrm{mg}$ of the crushed freeze-dried cured geopolymer was immediately analyzed by thermogravimetry (TA SDT-Q600) in order to calculate the residual water. The weight loss was measured over a range of temperature of $(30-950){ }^{\circ} \mathrm{C}$ at a heating rate of $20^{\circ} \mathrm{C} / \mathrm{min}$. Nitrogen was used as purging gas with a flow rate of $50 \mathrm{~mL} / \mathrm{min}$. The quantity of free water was calculated from the mass difference in the cured geopolymer before and 
after lyophilization. The quantity of bound water was determined by interpreting the TGA curve of the samples. Reported results are the average value of three samples.

\section{c. Thermoporometry}

Thermoporometry uses the heat dissipated due to the melting or solidification of confined water, measured by DSC, to calculate the pore size distribution (PSD) of a porous material [27,28]. The working principle of TMP is recalled here for reference but more details can be found elsewhere $[13,29,30]$ :

i. While cooling down a saturated porous material, water confined in pores will freeze at a temperature $T_{s}$ which is lower than the freezing temperature of bulk water $T_{0}$. The temperature shift, $\Delta T=T_{s}-T_{0}$, is inversely proportional to the pore size according to a relationship derived from the Kelvin-Laplace equation [28], for cylindrical ice crystal:

$$
\frac{T_{s}-T_{0}}{T_{0}}=\frac{2 \gamma_{l w} v_{l}}{r_{p} \Delta H_{f}}
$$

where $\gamma_{l w}$ is the liquid/wall interface tension, $v_{l}$ is the molar volume of water, $r_{p}$ is the pore radius and $\Delta H_{f}$ is the crystallization enthalpy. If the pore is cylindrical, this can be reduced to the following numerical equation [27]:

$$
r_{p}=\frac{-32.33}{\Delta T}+0.68
$$

ii. Over a temperature interval, the volume of pores in which the phase transition of confinement water takes place is obtained by dividing the heat release, measured 
during melting or solidification, by the corresponding (melting or solidification) enthalpy and ice density $\rho_{\text {ice }}$ :

$$
\Delta V=\frac{\Delta Q}{\Delta H} \frac{1}{\rho_{\text {ice }}}
$$

Using the two previous relations allows for the determination of the pore volume corresponding to a given pore size, thus enabling the determination of the pore size distribution (assuming a cylindrical pore shape). The detailed theory of thermoporometry, as well as its reviews and potential use in the field of civil engineering can be found in $[27,30,31]$. Thermoporometry appears superior to the conventional characterization methods (such as nitrogen adsorption, mercury intrusion porosimetry, and imaging by scanning electron microscopy) in that it does not require a drying procedure. But in practice, TMP is used less than other methods based on capillary condensation due to some difficulties in interpretation, especially the impact of ionic species on the freezing point depression $[29,30,25,32]$. This drawback was avoided here thanks to the stoppage involving exchange of the pore solution with pure water as discussed previously.

The DSC was performed on a TA Differential Scanning Calorimeter (TA Q200), using liquid nitrogen as refrigerating liquid. About $20 \mathrm{mg}$ of washed geopolymer was put on an aluminum hermetic pan $\left(\right.$ Tzero $\left.^{\circledR}\right)$ then sealed with an aluminum lid. We used a specific thermal procedure in order to prevent liquid water supercooling and isolate the macropore region inside the porous materials, as recommended in references $[13,30,33,34]$. The procedure consists of four sequences (Fig. 1). 1) A flash cooling sequence down to $-50^{\circ} \mathrm{C}$, freezing all the water in the sample. 2) A first heating sequence at $5{ }^{\circ} \mathrm{C} / \mathrm{min}$ up to $-0.2^{\circ} \mathrm{C}$, as recommended in [25], during which ice in the pore below $325 \mathrm{~nm}$ (cf. Eq. (2)) melted, while ice in the pore above $325 \mathrm{~nm}$ still persisted and provided nucleation site for the next cooling sequence. 3) A cooling sequence down to $-50{ }^{\circ} \mathrm{C}$ during which water froze again but this time without possibility of supercooling due to the 
persistence of ice in the macropores. 4) Finally, a second heating to the room temperature was performed to totally melt the ice in the bulk.

\section{d. Nitrogen adsorption, pycnometry and X-Ray diffraction}

The specific area and the pore size distribution of the sodium geopolymer samples were measured by nitrogen gas adsorption for the sake of comparison with DSC. About $0.3 \mathrm{~g}$ of washed geopolymer was degassed and dried under vacuum at $80{ }^{\circ} \mathrm{C}$ for $15 \mathrm{~h}$ before being analyzed (Micromeritics Tristar II 3020). The specific surface area was calculated from the adsorption isotherm using the BET method over a relative pressure range of 0.01 to 0.26 . The pore size distribution was calculated using the BJH method also from the adsorption isotherms.

The bulk density and porosity of sample were determined using a liquid pycnometer. Samples were crushed into small pieces a few millimeters in size. Water was the filling liquid and entrapped air removal (deairing) was performed with a dry primary vacuum pump.

XRD patterns were collected using a Philips PW 1700 X-ray diffractometer with $\mathrm{CuK}_{\alpha}$ radiation, a graphite monochromator, and operated in $\theta-2 \theta$ geometry at $40 \mathrm{kV}, 40 \mathrm{~mA}$. A $2 \theta$ range of $10^{\circ}$ to $100^{\circ}$ was explored with a step size of $0.025^{\circ}$ at a scanning rate of $0.25 \% \mathrm{~min}$.

\section{Results}

Thermoporometry is based on the phase transition of water inside a saturated porous sample. Therefore, it is important to know the state of water before any further investigation on this procedure. In particular, it is necessary to distinguish and quantify in the pores the relative amounts of freezing and non-freezing water. 


\section{State of water}

The total mass of the sample, $m_{\text {total }}$, can be distributed between the solid, $m_{s}$, the free water removable by freeze drying, $m_{w}^{f}$, and the residual water, $m_{w}^{r}$ (Fig. 2):

$$
m_{\text {total }}=m_{s}+m_{w}^{f}+m_{w}^{r}
$$

The initial total fraction of water is simply the water content of the raw materials:

$$
\phi_{T}=\left(m_{w}^{f}+m_{w}^{r}\right) / m_{\text {total }}
$$

For the cured geopolymer under consideration, it can be estimated from its formulation to be 35.8 wt $\%$ (36.4 wt $\%$ when considering $\mathrm{NaOH}$ as $\mathrm{Na}_{2} \mathrm{O} . \mathrm{H}_{2} \mathrm{O}$ ). The amount of free moisture of Metamax, $0.5 \mathrm{wt} \%$ as derived from a TGA, was taken into account in the calculation above.

From the difference in mass before, $m_{\text {total }}$, and after, $m_{\text {lyophilized }}$, freeze-drying, one gets the fraction of free water:

$$
\phi_{1}=\frac{m_{\text {total }}-m_{\text {lyophilized }}}{m_{\text {total }}}=\frac{m_{w}^{f}}{m_{\text {total }}}
$$

The residual water fraction $\phi_{2}$ in the lyophilized sample was determined from the weight loss of the lyophilized samples in a TGA experiments:

$$
\phi_{2}=\frac{m_{w}^{r}}{m_{\text {lyophilized }}}
$$

From that, one can get the faction of residual water in the sample:

$$
\phi_{3}=\frac{m_{w}^{r}}{m_{\text {total }}}=\left(1-\phi_{1}\right) \phi_{2}
$$

The weight loss and the corresponding differential thermogravimetry (DTG) curves for all cured sodium geopolymer pastes after freeze drying at different ages are represented in Fig. 3 . The 
weight loss began approximately at $50{ }^{\circ} \mathrm{C}$ and ended at $600{ }^{\circ} \mathrm{C}$. For all samples, the weight loss peak was centered at $140{ }^{\circ} \mathrm{C}$ and the cutoff was fixed at $420{ }^{\circ} \mathrm{C}$ (further registered weight loss inferior to $5 \%$ of the total registered weight loss).

The results of these simple mass balance considerations are given in Table 1. Noticeably, the total water mass balance was constant within $0.6 \mathrm{wt} \%$ and remarkably close to the nominal value of $36.4 \mathrm{wt} \%$ expected from the water content of the raw materials. Retrospectively, this confirmed the good quality of the procedure and the fact that no water was lost. It must also be noted that free water represented a large majority $(\sim 73 \mathrm{wt} \%)$ of the water initially present. The residual water may have consisted in chemically bound water (silanol groups for example) but more importantly in the adsorbed layers of non-freezing water, as described in the literature [35]. To verify the fact that the residual water after freeze-drying was indeed a non-freezing water layer, lyophilized samples were subjected to DSC according to the thermal procedure described in Fig. 1. To minimize any influence of air moisture, all lyophilized sample were submitted to DSC immediately after the freeze-drying process. Figures S1-S4 represent the thermogram of lyophilized samples - the $1^{\text {st }}$ cooling sequence is not included in the curve because the flash cooling only serves to freeze all possible water (bulk + confinement water).

No sign of phase transition, fusion or solidification could be detected on thermograms of freeze-dried cured sodium geopolymers whatever their age (see Supplemental Materials). This simple test further demonstrated that the residual water was indeed non-freezing. The thermogram of lyophilized cured samples could thus be used as baseline correction for the DSC test of saturated washed sodium geopolymer pastes in order to isolate the contribution of free water. 


\section{Validation of the DSC procedure}

First, in order to verify the reliability of the DSC apparatus, a preliminary test on Milli-Q water was performed on our DSC machine. Water was cooled down to $-40{ }^{\circ} \mathrm{C}$ then heated up to room temperature at the rate mentioned in Fig. $1\left(5{ }^{\circ} \mathrm{C} / \mathrm{min}\right)$. The melting enthalpy of Milli-Q water, derived from the heating sequence, was equal to $326.1 \mathrm{~J} / \mathrm{g}$. This experimental value is only $2 \%$ lower than the standard value for water $(333.4 \mathrm{~J} / \mathrm{g})$ and thus provides an estimate of the accuracy. Moreover, on each sample, the heat flow curves for the first and second heating sequences of the DSC protocol described in the experimental section (Fig. 4) were identical, reflecting the precision of the experiments. Furthermore, this meant that the thermal rate $\left(5^{\circ} \mathrm{C} / \mathrm{min}\right)$ preserved the internal pore structure of the sample.

Next, to justify the washing procedure, the different DSC behavior of the as-synthesized pore solution and free water was highlighted. For that purpose, the thermogram of a cured sample $(168 \mathrm{~h})$ and of the same sample after the exchange of the pore solution (that is, a washed sample according to the stoppage and solution exchange procedure described in the experimental section) were compared (Fig. 5).

The first endothermic peaks $\left(T \sim-8.5^{\circ} \mathrm{C}\right)$ of the heating sequences of the two samples were at the same position but the second one was narrower and shifted to lower temperatures $\left(3.2^{\circ} \mathrm{C}\right.$, in comparison to $5.5{ }^{\circ} \mathrm{C}$ ) after washing the sample. Similarly, the exothermic peaks in the cooling processes shifted to higher temperatures (by $3-6{ }^{\circ} \mathrm{C}$ ) after washing. These shifts were likely to be due to the difference in composition of the pore solution versus pure water. The washing procedure was thus essential for a correct interpretation of the DSC results in the context of thermoporometry [27]. This is because the numerical values of the parameters in Eq.(2) linking temperature shifts and pore sizes are calibrated using reference porous samples saturated by pure 
water. For example, using Eq.(2), the 3 peaks in the cured sample $\left(-5.1{ }^{\circ} \mathrm{C},-36.8{ }^{\circ} \mathrm{C}\right.$ and $\left.-44^{\circ} \mathrm{C}\right)$ would lead to an underestimation of the pore diameters (14 nm, $3.1 \mathrm{~nm}$ and $2.8 \mathrm{~nm}$, respectively). In fact, replacing the pore solution with water as it occurs in the washed samples revealed that after $168 \mathrm{~h}$ of curing time, the true pore diameters were $35 \mathrm{~nm}\left(-1.9^{\circ} \mathrm{C}\right), 3.5 \mathrm{~nm}\left(-30^{\circ} \mathrm{C}\right)$ and $3 \mathrm{~nm}(-$ $39.8^{\circ} \mathrm{C}$ ). Not washing the samples would thus have led to significant errors in the mesopore region.

\section{Determination of the pore size distribution by low temperature DSC}

By subtracting the heat flow recorded on the washed sample to the one obtained on the lyophilized sample (which serves as a baseline), the heat flow due to the contribution of residual water was eliminated and the one due to water confined in pores was isolated (Fig. 5). This was confirmed as follows. The total energy of phase transition of water confined during the $1^{\text {st }}$ heating and $2^{\text {nd }}$ cooling sequences (Fig. 5), in absolute value, were calculated by numerical integration. The relative error between the heats of phase transition of the two thermal consequences was also calculated and is shown in Table 2. The energy balance between the two consecutive sequences is very close to zero, thus validating the usage of the thermogram of the lyophilized sample as a baseline.

Once the contribution of the solid and of the residual water was eliminated by baseline substraction, the DSC thermogram could be interpreted in terms of thermoporetry. As the phase transition energy is proportional to the quantity of confined water, the heat measured in DSC is actually a measurement of the same state variable as adsorption measurements, namely the quantity of water molecules. Considering that phase solidification/melting temperature and gas condensation are both functions of the pore size, the plot of the cumulative energy versus the temperature is conceptually analogous to an adsorption isotherm where pressure replaces temperature as the state variable. The analogy is not direct though as thermoporometry is based on 
the phase transition of confined water occurring within the pores themselves without exchange with the outside whereas nitrogen gas adsorption is a pore filling process. A comparison between the two mechanism on a model mesoporous material has been reported in [25]. Considering that the path followed by the advancing liquid/solid interface in thermoporometry and by the liquid/gas one in gas adsorption is the same, similar hysteresis loops should be observed by the two methods. Along this line, Fig. 7 represents the cumulative energy associated to the water phase transition in the pores of the sodium geopolymers in comparison with their corresponding normalized adsorption-desorption isotherm at different curing times.

\section{IV.Discussion}

As expected, both porosimetry methods, DSC, and nitrogen adsorption isotherms presented similar hysteretic behavior (Figure 6). As the geopolymerization time evolved, the hysteresis loops began at lower temperature or relative pressure $\left(\sim-6^{\circ} \mathrm{C}\right.$ and $\sim 0.85$ respectively). In the isotherms, the closure of the hysteresis loop occurred around the relative pressure of 0.4 for all ages, whereas in thermoporometry it happened at lower temperature as time evolved. Before the capillary condensation leading to the hysteresis, nitrogen gas sorption showed a gas adsorption pattern at low relative pressure due to molecular gas adsorption (i.e. BET multilayer adsorption). TPM did not show this pattern because of the presence of a non-freezing residual water layer at the surface.

The pore size distribution obtained from the two methods assuming cylindrical pores are shown in Fig. 7. The nitrogen adsorption/desorption isotherms were analyzed using the BJH method and the cumulative DSC energy using equations (2) \& (3).

The mesopore volume measured by the two methods (that is, by integrating the PSD up to $50 \mathrm{~nm}$ ) were compared with the total pore volume (in $\mathrm{mL} / \mathrm{g}$ ) measured using a liquid pycnometer 
(Table 3). Regardless of the method, TPM or NAP, the measured volume of mesopores decreased with curing time just like the total pore volume fraction measured by pycnometry did. However, thermoporometry (TPM) captured more mesoporous volume than nitrogen gas adsorption $(\sim 1.5$ times higher at every specific age). This reflected the fact that the non-invasive sample preparation of TPM better preserved the pore structure of the samples. TPM can also quantify the amount of macropores in the materials, which would not be accessible while using nitrogen gas adsorption. Note that the porous volume and average pore diameters observed here by gas adsorption was very close to the one observed for the same formulation after one month by Benavent et al [12].

Concerning the pore size distribution, a similar trend was observed by the two methods: the pores were finer as the curing time increased. However, this evolution was more pronounced when using TMP; again probably because this method preserved better the pore structure. The fact that the pore structure evolved was expected as calorimetry data [36] clearly shows that the geopolymerization still proceeds after $24 \mathrm{~h}$. The data obtained here by TPM in unconsolidated pastes aged for less than a week nicely completed already published works on the structural evolution of consolidated geopolymer pastes between seven days and a month [36]. Before one week, in the unconsolidated paste, polycondensation still proceeded from the solution resulting in a building of the pore structure and a decrease of the mesopore sizes. After that time, Benavent et al. [12] have shown that the pore solution composition is essentially constant. The consolidated pore structure still reacts but essentially by reorganization of the aluminosilica network, resulting in some pore closure and an apparent coarsening of the mesopores available for nitrogen adsoption.

The evolution of the BET specific area was also evaluated from the isotherms (Fig. 8). The specific surface area varied only slightly around $70 \mathrm{~m}^{2} \cdot \mathrm{g}^{-1}$, reflecting the concomitant diminutions of the mesopore volume and of the pore sizes, one effect compensating the other. From this value, 
it is interesting to question the nature of the residual water. A first hypothesis would be that it corresponds to strongly bound water on the geopolymer surface. Considering that the total fraction of water $\phi_{T}$ was $36.4 \mathrm{wt} \%$ and taking a fraction of residual water $\phi_{3}$ of about 0.1 , the ratio of residual water to the solid fraction was about $\left(1-\phi_{T}\right) \phi_{3} \approx 0.16$. For a specific surface area of 70 $\mathrm{m}^{2} \cdot \mathrm{g}^{-1}$, if a water molecule occupies a surface area of approximately $11.410^{-2} \mathrm{~nm}^{2}$, this would lead to consider that the residual water corresponded to about an eight-molecule thick layer. This is clearly unrealistic and this hypothesis must be discarded. Residual water did not correspond either to silanol groups as they represented only $3 \%$ of the intensity of the total magic angle spinning ${ }^{1} \mathrm{H}$ nuclear magnetic resonances in the lyophilized samples (Fig. 9). A more probable explanation would be that this residual water corresponded to water molecules tightly bound in the hydration shell of the sodium counter ions. Indeed, from the chemical composition of the solid sodium geopolymer, one can calculate that the residual water amounts for about $1.6 \mathrm{H}_{2} \mathrm{O}$ per $\mathrm{Na}^{+}$. This is astonishingly close the 1.3 ratio encountered in hydrosodalite, a crystalline zeolite of composition very close to amorphous geopolymer and sometimes used to model their local range order [37,38].

\section{Summary and conclusions}

In this study, we have used DSC to analyze the state of water and quantify the pore size distribution by TPM in typical metakaolin-based fresh geopolymer pastes less than a week old. This was made possible by an adapted stopping and washing procedure, and by building an experimental DSC baseline that takes into account the impact of residual non-freezing water layer. The characterization by TPM of the evolution of the microstructure of sodium-based geopolymer revealed two important facts consistent with NAP results: 
1. While the total porous fraction remained roughly constant, the mesopore volume decreased during the curing time (Table 3).

2. Simultaneously, a refinement of the pore system, or at least of its mesoporous fraction, was observed as the pore size distribution shifted towards the micropore region (Fig. 7).

The instability of the porous structure before one month of curing has already been reported [12]. However, the ability of TPM to characterize unconsolidated materials shed new light on the complex temporal evolution of the structure of geopolymer pastes. NAP showed a sudden shift of the mean pore size from about $20 \mathrm{~nm}$ to $8-9 \mathrm{~nm}$ after one day. However, the pore structure of the unconsolidated geopolymer is unlikely to remain constant during the drying step necessary for NAP and consequently this shift could be questioned. In contrast to NAP, TPM directly quantifies the amount of water filling the pores and consequently is more likely to respect the pore structure before consolidation. TPM thus unveiled that the reduction in pore size was actually gradual over the same time scale. Consequently, TPM revealed that, prior to the pore coarsening previously observed by nitrogen adsorption in consolidated geopolymers, in the very early age (less than a week) of the unconsolidated material, the initial pore structure actually refined continuously during geopolymerization. This was probably due to the fact that the geopolymer network was still actively condensing from the activation solution.

Finally, from mass balance consideration, the residual water was identified with the hydration shell of the sodium counter ions with only a minor occurrence of covalently bound protons as silanol groups. 


\section{Acknowledgments}

QHN acknowledges the financial support of BASF Construction Solutions during his postdoctoral work. The help of Ilya V. Yakovlev (SIMM, ESPCI Paris) and Farid Nouar (IMAP, ESPCI Paris) for solid-state NMR and nitrogen adsorption experiments, respectively, are gratefully acknowledged.

\section{References}

[1] G. Habert, J.B. d'Espinose de Lacaillerie, N. Roussel, An environmental evaluation of geopolymer based concrete production: reviewing current research trends, J. Clean. Prod. 19 (2011) 1229-1238. https://doi.org/10.1016/j.jclepro.2011.03.012.

[2] G.P. Kutyla, W.M. Kriven, Properties and Characterization of Alumina Platelet Reinforced Geopolymer Composites, J. Am. Ceram. Soc. (n.d.).

[3] V. Cantarel, M. Arisaka, I. Yamagishi, On the hydrogen production of geopolymer wasteforms under irradiation, J. Am. Ceram. Soc. 102 (2019) 7553-7563.

[4] S. Petlitckaia, A. Poulesquen, Design of lightweight metakaolin based geopolymer foamed with hydrogen peroxide, Ceram. Int. 45 (2019) 1322-1330.

[5] Y. Luna Galiano, C. Fernández Pereira, J. Vale, Stabilization/solidification of a municipal solid waste incineration residue using fly ash-based geopolymers, J. Hazard. Mater. 185 (2011) 373-381.

[6] A.J.G. Bourlon, O. Porcherie, S.L. Roy-Delage, Compositions and methods for cementing wells, US20170306214A1, 2017.

[7] P. Duxson, J.L. Provis, G.C. Lukey, S.W. Mallicoat, W.M. Kriven, J.S. Van Deventer, Understanding the relationship between geopolymer composition, microstructure and mechanical properties, Colloids Surf. Physicochem. Eng. Asp. 269 (2005) 47-58.

[8] W.M. Kriven, J.L. Bell, M. Gordon, Microstructure and Microchemistry of Fully-Reacted Geopolymers and Geopolymer Matrix Composites, in: Adv. Ceram. Matrix Compos. IX, John Wiley \& Sons, Ltd, 2012: pp. 227-250.

[9] C. Boher, I. Martin, S. Lorente, F. Frizon, Experimental investigation of gas diffusion through monomodal materials. Application to geopolymers and Vycor® glasses, Microporous Mesoporous Mater. 184 (2014) 28-36.

[10] R. Pouhet, M. Cyr, R. Bucher, Influence of the initial water content in flash calcined metakaolin-based geopolymer, Constr. Build. Mater. 201 (2019) 421-429.

[11] C.F. Maitland, C.E. Buckley, B.H. O'connor, P.D. Butler, R.D. Hart, Characterization of the pore structure of metakaolin-derived geopolymers by neutron scattering and electron microscopy, J. Appl. Crystallogr. 44 (2011) 697-707.

[12] V. Benavent, F. Frizon, A. Poulesquen, Effect of composition and aging on the porous structure of metakaolin-based geopolymers, J. Appl. Crystallogr. 49 (2016) 2116-2128. 
[13] Q.H. Nguyen, S. Lorente, A. Duhart-Barone, H. Lamotte, Porous arrangement and transport properties of geopolymers, Constr. Build. Mater. 191 (2018) 853-865.

[14] I. Ismail, S.A. Bernal, J.L. Provis, S. Hamdan, J.S.J. van Deventer, Drying-induced changes in the structure of alkali-activated pastes, J. Mater. Sci. 48 (2013) 3566-3577.

[15] B.J. Christensen, T. Coverdale, R.A. Olson, S.J. Ford, E.J. Garboczi, H.M. Jennings, T.O. Mason, Impedance Spectroscopy of Hydrating Cement-Based Materials: Measurement, Interpretation, and Application, J. Am. Ceram. Soc. 77 (1994) 2789-2804.

[16] J. Zhang, G.W. Scherer, Comparison of methods for arresting hydration of cement, Cem. Concr. Res. 41 (2011) 1024-1036.

[17] R. Snellings, J. Chwast, Ö. Cizer, N. De Belie, Y. Dhandapani, P. Durdzinski, J. Elsen, J. Haufe, D. Hooton, C. Patapy, Report of TC 238-SCM: hydration stoppage methods for phase assemblage studies of blended cements - results of a round robin test, Mater. Struct. 51 (2018) 111.

[18] X. Chen, A. Meawad, L.J. Struble, Method to stop geopolymer reaction, J. Am. Ceram. Soc. 97 (2014) 3270-3275.

[19] P. Rovnaník, Effect of curing temperature on the development of hard structure of metakaolin-based geopolymer, Constr. Build. Mater. 24 (2010) 1176-1183.

[20] P. Steins, A. Poulesquen, F. Frizon, O. Diat, J. Jestin, J. Causse, D. Lambertin, S. Rossignol, Effect of aging and alkali activator on the porous structure of a geopolymer, J. Appl. Crystallogr. 47 (2014) 316-324.

[21] P. Steins, A. Poulesquen, O. Diat, F. Frizon, Structural evolution during geopolymerization from an early age to consolidated material, Langmuir. 28 (2012) 8502-8510.

[22] A. Favier, J. Hot, G. Habert, N. Roussel, J.-B. d'Espinose de Lacaillerie, Flow properties of MK-based geopolymer pastes. A comparative study with standard Portland cement pastes, Soft Matter. 10 (2014) 1134-1141.

[23] Y. Malier, High Performance Concrete: From material to structure, CRC Press, 1992.

[24] F.D. Larrard, Concrete Mixture Proportioning: A Scientific Approach, CRC Press, 1999.

[25] I. Beurroies, R. Denoyel, P. Llewellyn, J. Rouquerol, A comparison between meltingsolidification and capillary condensation hysteresis in mesoporous materials: application to the interpretation of thermoporometry data, Thermochim. Acta. 421 (2004) 11-18.

[26] R.R. Lloyd, J.L. Provis, J.S. Van Deventer, Pore solution composition and alkali diffusion in inorganic polymer cement, Cem. Concr. Res. 40 (2010) 1386-1392.

[27] M. Brun, A. Lallemand, J.-F. Quinson, C. Eyraud, A new method for the simultaneous determination of the size and shape of pores: the thermoporometry, Thermochim. Acta. 21 (1977) 59-88.

[28] R. Denoyel, R.J.M. Pellenq, Simple phenomenological models for phase transitions in a confined geometry. 1: Melting and solidification in a cylindrical pore, Langmuir. 18 (2002) 2710-2716.

[29] C. Eyraud, J.F. Quinson, M. Brun, The role of thermoporometry in the study of porous solids, in: Stud. Surf. Sci. Catal., Elsevier, 1988: pp. 295-305.

[30] Z. Sun, G.W. Scherer, Pore size and shape in mortar by thermoporometry, Cem. Concr. Res. 40 (2010) 740-751.

[31] M.R. Landry, Thermoporometry by differential scanning calorimetry: experimental considerations and applications, Thermochim. Acta. 433 (2005) 27-50.

[32] A.M. Kjeldsen, M.R. Geiker, On the interpretation of low temperature calorimetry data, Mater. Struct. 41 (2007) 213-224. 
[33] J.F. Quinson, M. Brun, Progress in thermoporometry, in: Stud. Surf. Sci. Catal., Elsevier, 1988: pp. 307-315.

[34] S. Irico, D. Gastaldi, F. Canonico, G. Magnacca, Investigation of the microstructural evolution of calcium sulfoaluminate cements by thermoporometry, Cem. Concr. Res. 53 (2013) 239-247.

[35] D.S. Perera, E.R. Vance, K.S. Finnie, M.G. Blackford, J.V. Hanna, D.J. Cassidy, Disposition of water in metakaolinite based geopolymers, Adv. Ceram. Matrix Compos. XI. 175 (2006) 225-236.

[36] J. Aupoil, J.-B. Champenois, J.-B. d'Espinose de Lacaillerie, A. Poulesquen, Interplay between silicate and hydroxide ions during geopolymerization, Cem. Concr. Res. 115 (2019) 426-432. https://doi.org/10.1016/j.cemconres.2018.09.012.

[37] C.E. White, K. Page, N.J. Henson, J.L. Provis, In situ synchrotron X-ray pair distribution function analysis of the early stages of gel formation in metakaolin-based geopolymers, Appl. Clay Sci. 73 (2013) 17-25.

[38] T. Williamson, L.E. Katz, J. Han, H.A. Dobbs, B.F. Chmelka, G. Sant, M.C. Juenger, Relationship between aqueous chemistry and composition, structure, and solubility of sodium aluminosilicate hydrates, J. Am. Ceram. Soc. 103 (2020) 2160-2172. 


\section{FIGURE CAPTIONS}

Figure 1 : Schematic representation of the DSC thermal procedure. The purpose of the preparatory steps is to generate ice in the large macropores, thus providing nucleation sites at the entrance of the smaller pores and preventing supercooling of liquid water during the cooling step.

Figure 2 : State of water in porous sample, before (a) and after (b) freeze-drying.

Figure 3: Weight loss and DTG curve of freeze-dried cured sodium geopolymer samples at different curing times.

Figure 4 : Comparison of the DSC thermogram of a cured sample and a washed sample at $168 \mathrm{~h}$ curing time. The first fast cooling is not represented as, due to supercooling, it carries no information.

Figure 5 : Thermogram of water confined in the pores of the sodium washed geopolymer during the $1^{\text {st }}$ heating sequence (a) and $2^{\text {nd }}$ cooling sequence (b), after subtracting the baseline.

Figure 6 : Normalized cumulative phase transition energy of water confined in the pores of washed sodium geopolymer at different stopping time (left) and the normalized nitrogen adsorptiondesorption isotherm of the same samples after drying (right). The cumulative energy dissipated during the heating/cooling sequences are normalized by the total energy (reported in Table 2) just 
as the quantity of gas adsorbed is normalized to full adsorption. The vertical lines mark the approximate beginning of the hysteresis loop.

Figure 7 : Pore size distribution of washed sodium geopolymer obtained by thermoporometry (second cooling sequence) and nitrogen gas adsorption as a function of curing times. Note that the pore size range of application of the $\mathrm{BJH}$ analysis for nitrogen adsorption method (BJH) is 2-50 $\mathrm{nm}$. In our case, the upper limit of thermoporometry is $325 \mathrm{~nm}$ since the cooling step starts at $-0.2^{\circ} \mathrm{C}$.

Figure 8 : Evolution of BET specific surface area obtained by nitrogen gas adsorption, as a function of curing times.

Figure $9:{ }^{1} \mathrm{H}$ NMR of $23 \mathrm{~h}$ lyophilized sodium geopolymer. The experimental spectrum (top trace) can be decomposed in two resonances, a minor one at $1.9 \mathrm{ppm}$ related to the protons of the silanol groups $(\mathrm{SiOH})$, and a dominant one at $6.2 \mathrm{ppm}$ attributed to the protons in the sodium hydration water (lower traces). 
Table 2: Heat of the phase transition measured due to water confined in the pores of the sodium geopolymer during the $1^{\text {st }}$ heating and $2^{\text {nd }}$ cooling sequences

\begin{tabular}{|c|c|c|c|}
\hline \multirow{2}{*}{} & \multicolumn{2}{|c|}{ Heat (J) } & $\Delta(\%)$ \\
\cline { 2 - 3 } & $1^{\text {st }}$ heating & $2^{\text {nd }}$ cooling & \\
\hline $23 \mathrm{~h}$ & 59.5 & -56.2 & 5.6 \\
\hline $48 \mathrm{~h}$ & 47.2 & -49.7 & 5.5 \\
\hline $72 \mathrm{~h}$ & 28.7 & -30.6 & 6.7 \\
\hline $168 \mathrm{~h}$ & 23.3 & -21.6 & 7.4 \\
\hline
\end{tabular}

\section{TABLES}

Table 1: Relative content of free, residual, and total water calculated from freeze-drying and TGA experiments in cured geopolymer pastes at different curing times.

\begin{tabular}{|c|c|c|c|}
\hline $\begin{array}{c}\text { Curing time } \\
(\mathrm{h})\end{array}$ & $\begin{array}{c}\text { Free water } \\
\phi_{1} \\
(\mathrm{wt} \%)\end{array}$ & $\begin{array}{c}\text { Residual water } \\
\phi_{3}\end{array}$ & $\begin{array}{c}\text { Total water } \\
\phi_{T}=\phi_{1}+\phi_{3} \\
(\mathrm{wt} \%)\end{array}$ \\
\hline $24 \mathrm{ht} \%)$ & 28.2 & 8.0 & $\mathbf{3 6 . 2}$ \\
\hline $48 \mathrm{~h}$ & 26.9 & 9.7 & $\mathbf{3 6 . 5}$ \\
\hline $72 \mathrm{~h}$ & 26.3 & 10.5 & $\mathbf{3 6 . 8}$ \\
\hline $168 \mathrm{~h}$ & 25.2 & 11.2 & $\mathbf{3 6 . 5}$ \\
\hline
\end{tabular}


Table 3: Bulk density and porosity of geopolymer determined by pycnometry, and proportion of mesopore volume obtained by each porosimetry methods (reported to the total open pore volume obtained by pycnometry).

\begin{tabular}{|c|c|c|c|c|}
\hline \multirow{2}{*}{ Times } & $\begin{array}{c}\text { Bulk } \\
\text { density }\end{array}$ & \multirow{2}{*}{$\begin{array}{c}\text { Porosity } \\
(\%)\end{array}$} & \multicolumn{2}{|c|}{$\mathrm{V}_{\text {mesopore }} \mathrm{V}_{\text {open pore }}(\%)$} \\
\cline { 4 - 5 } & $\left(\mathrm{cm}^{3} / \mathrm{g}\right)$ & & Thermoporometry & $\begin{array}{c}\text { Nitrogen gas } \\
\text { adsorption }\end{array}$ \\
\hline $23 \mathrm{~h}$ & 1.14 & 51.9 & 78.9 & 49.2 \\
\hline $48 \mathrm{~h}$ & 1.20 & 48.7 & 73.4 & 46.8 \\
\hline $72 \mathrm{~h}$ & 1.19 & 47.7 & 51.9 & 34.9 \\
\hline $168 \mathrm{~h}$ & 1.15 & 47.3 & 40.5 & 25.9 \\
\hline
\end{tabular}




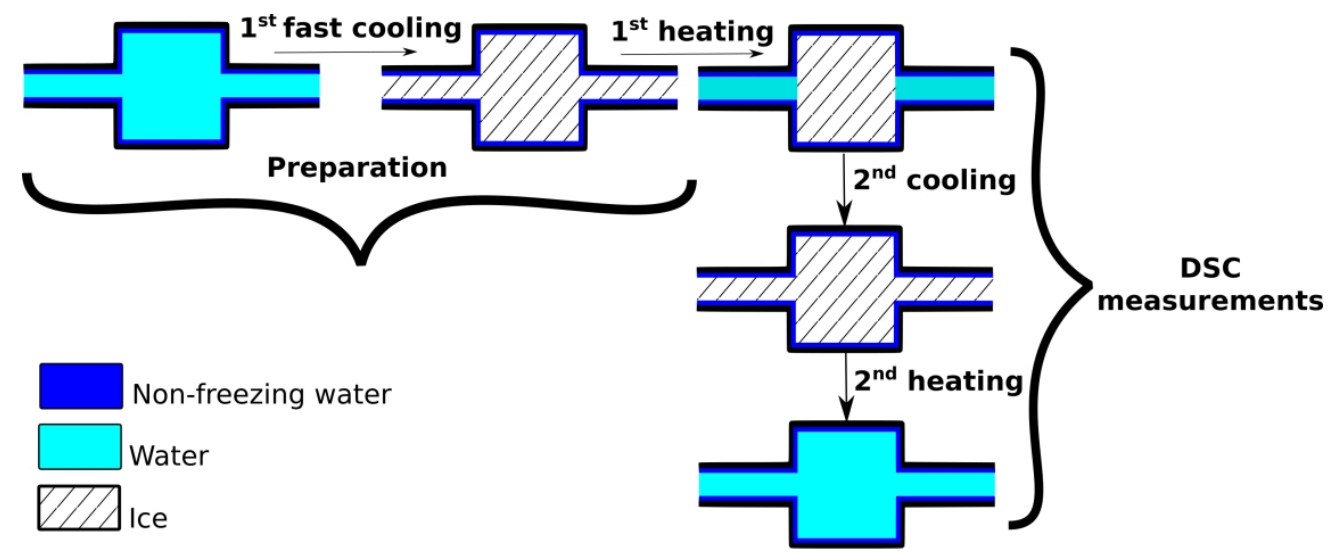

Figure 1 : Schematic representation of the DSC thermal procedure. The purpose of the preparatory steps is to generate ice in the large macropores, thus providing nucleation sites at the entrance of the smaller pores and preventing supercooling of liquid water during the cooling step. 

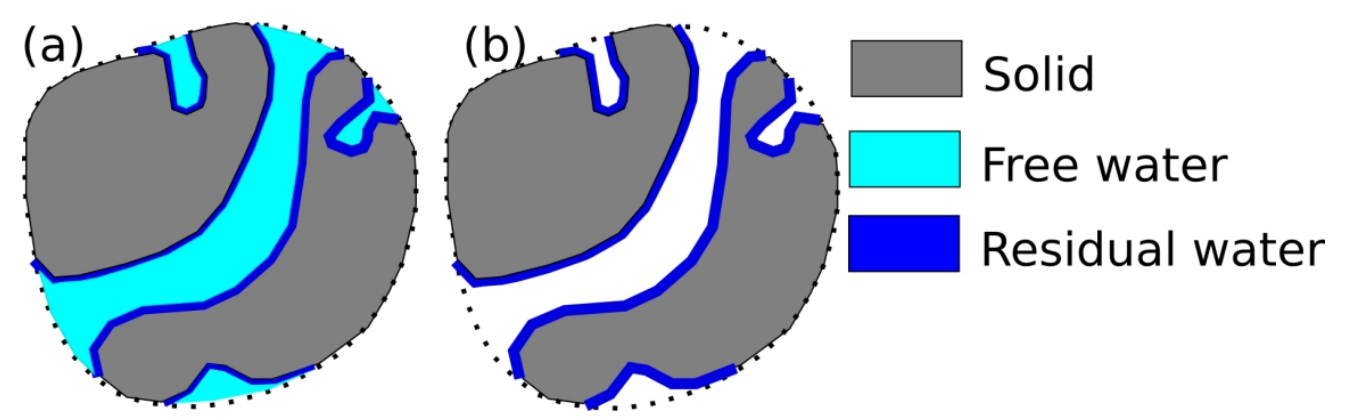

Figure 2 : State of water in porous sample, before (a) and after (b) freeze-drying. 


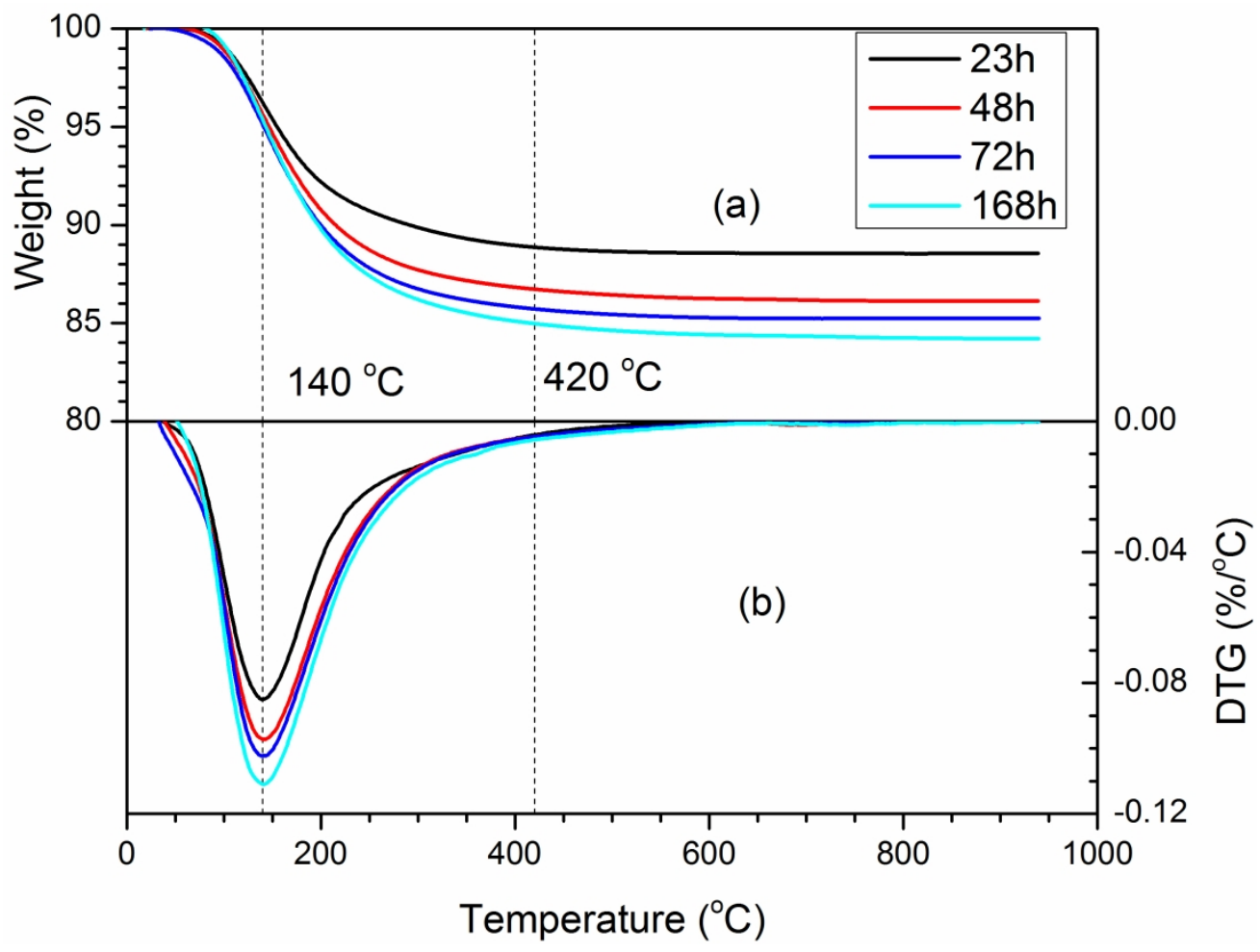

Figure 3 : Weight loss and DTG curve of freeze-dried cured sodium geopolymer samples at different curing times.

$245 \times 184 \mathrm{~mm}(300 \times 300$ DPI $)$ 


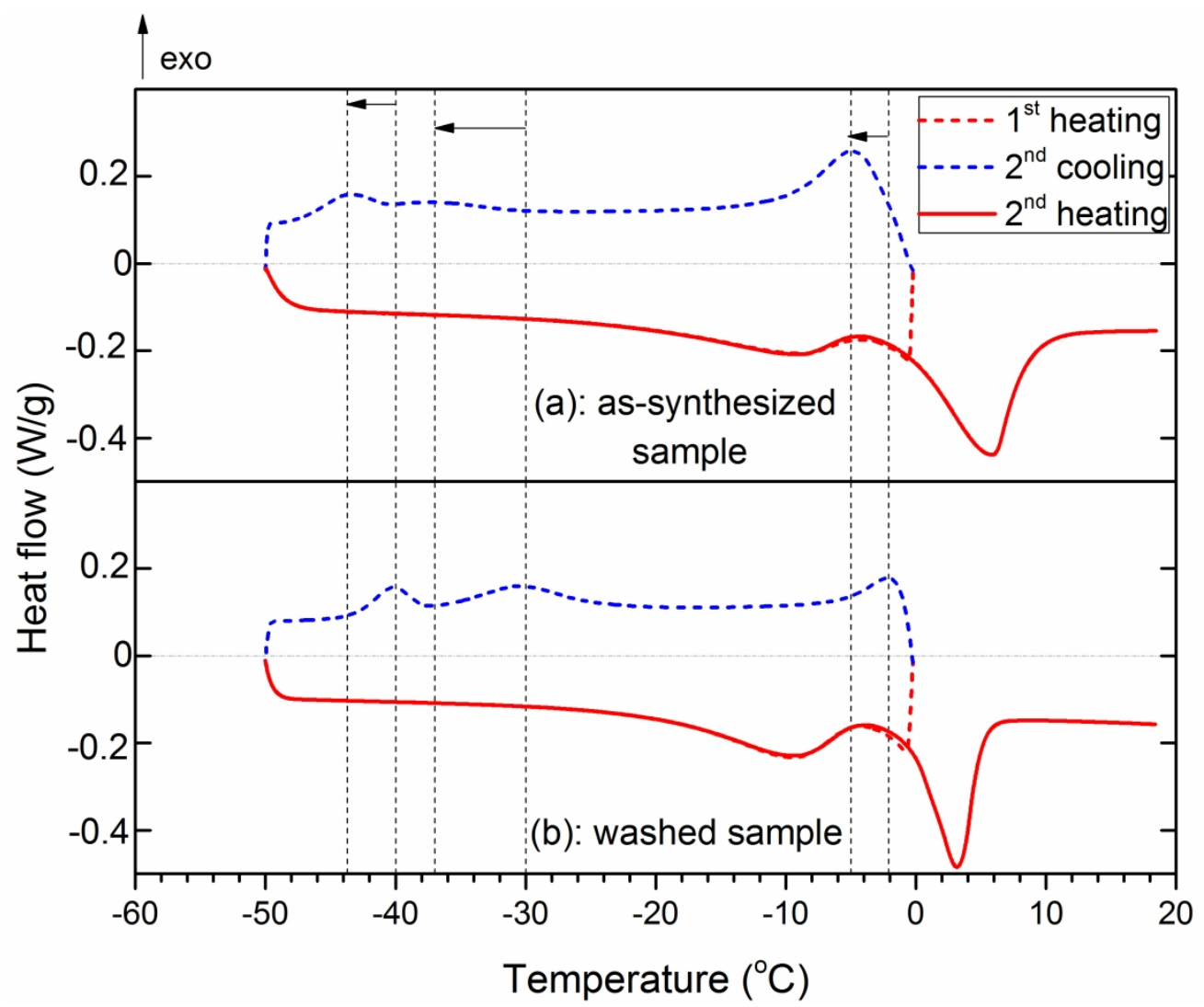

Figure 4 : Comparison of the DSC thermogram of a cured sample and a washed sample at $168 \mathrm{~h}$ curing time. The first fast cooling is not represented as, due to supercooling, it carries no information.

$234 \times 194 \mathrm{~mm}(300 \times 300$ DPI $)$ 


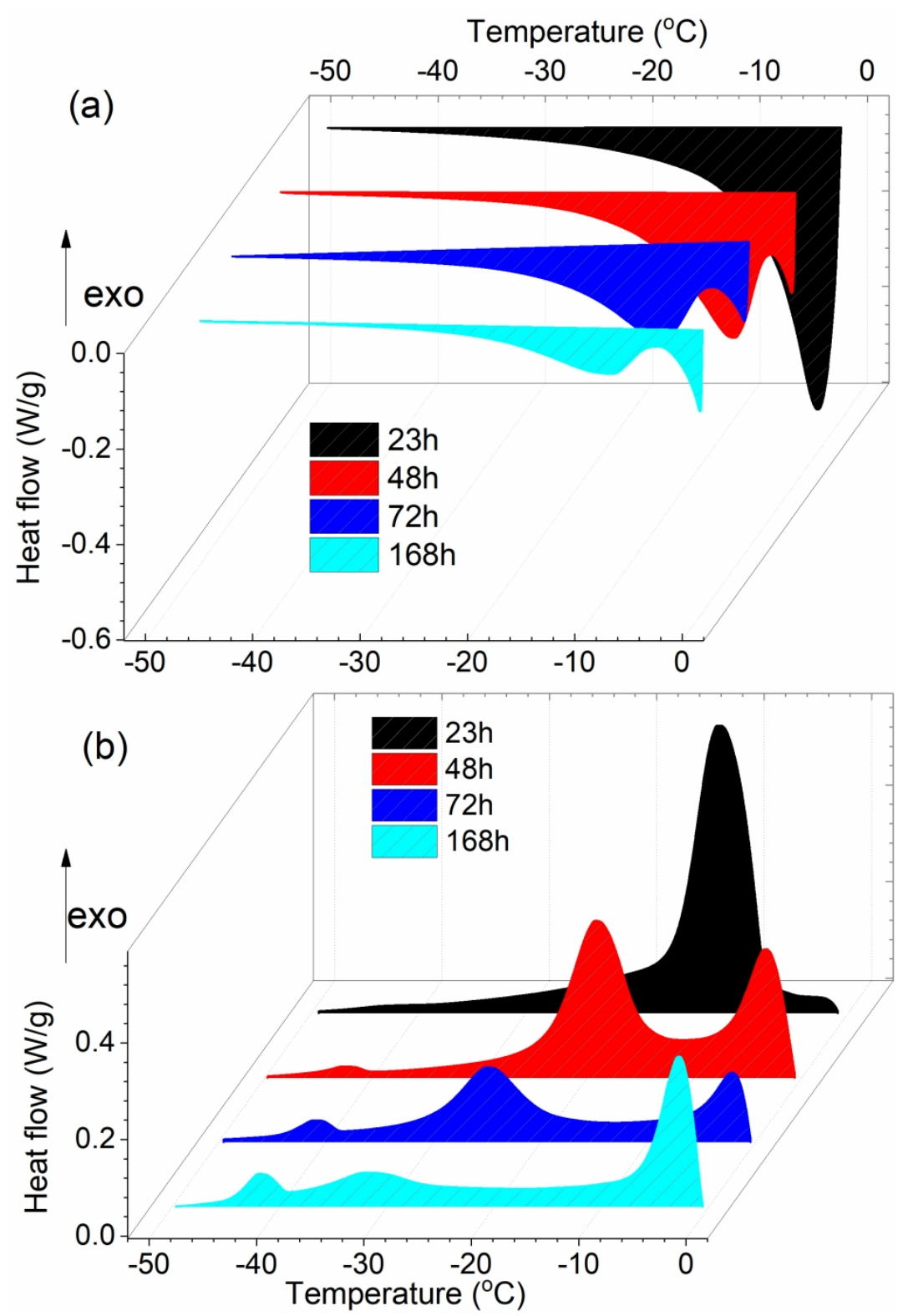

Figure 5 : Thermogram of water confined in the pores of the sodium washed geopolymer during the 1 st heating sequence (a) and 2nd cooling sequence (b), after subtracting the baseline.

$181 \times 266 \mathrm{~mm}(300 \times 300 \mathrm{DPI})$ 
(a)

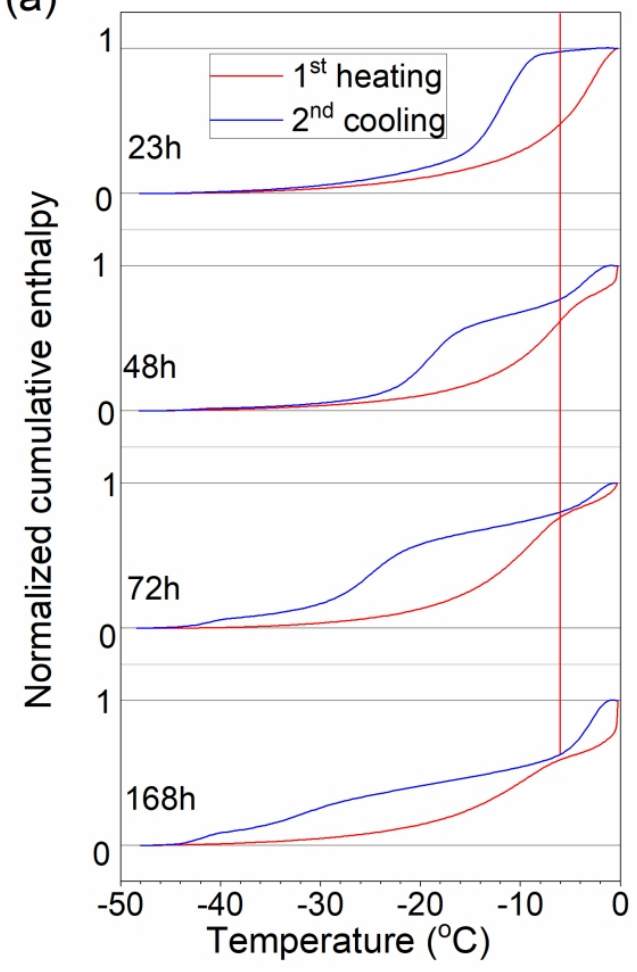

(b)

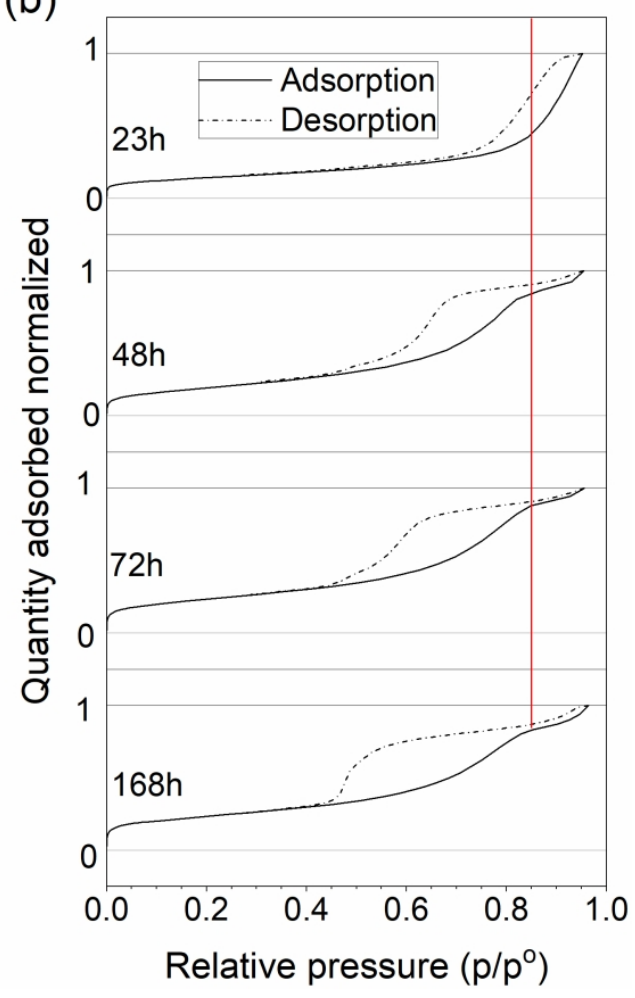

Figure 6 : Normalized cumulative phase transition energy of water confined in the pores of washed sodium geopolymer at different stopping time (left) and the normalized nitrogen adsorption-desorption isotherm of the same samples after drying (right). The cumulative energy dissipated during the heating/cooling sequences are normalized by the total energy (reported in Table 2) just as the quantity of gas adsorbed is normalized to full adsorption. The vertical lines mark the approximate beginning of the hysteresis loop.

$$
232 \times 182 \mathrm{~mm}(300 \times 300 \text { DPI })
$$




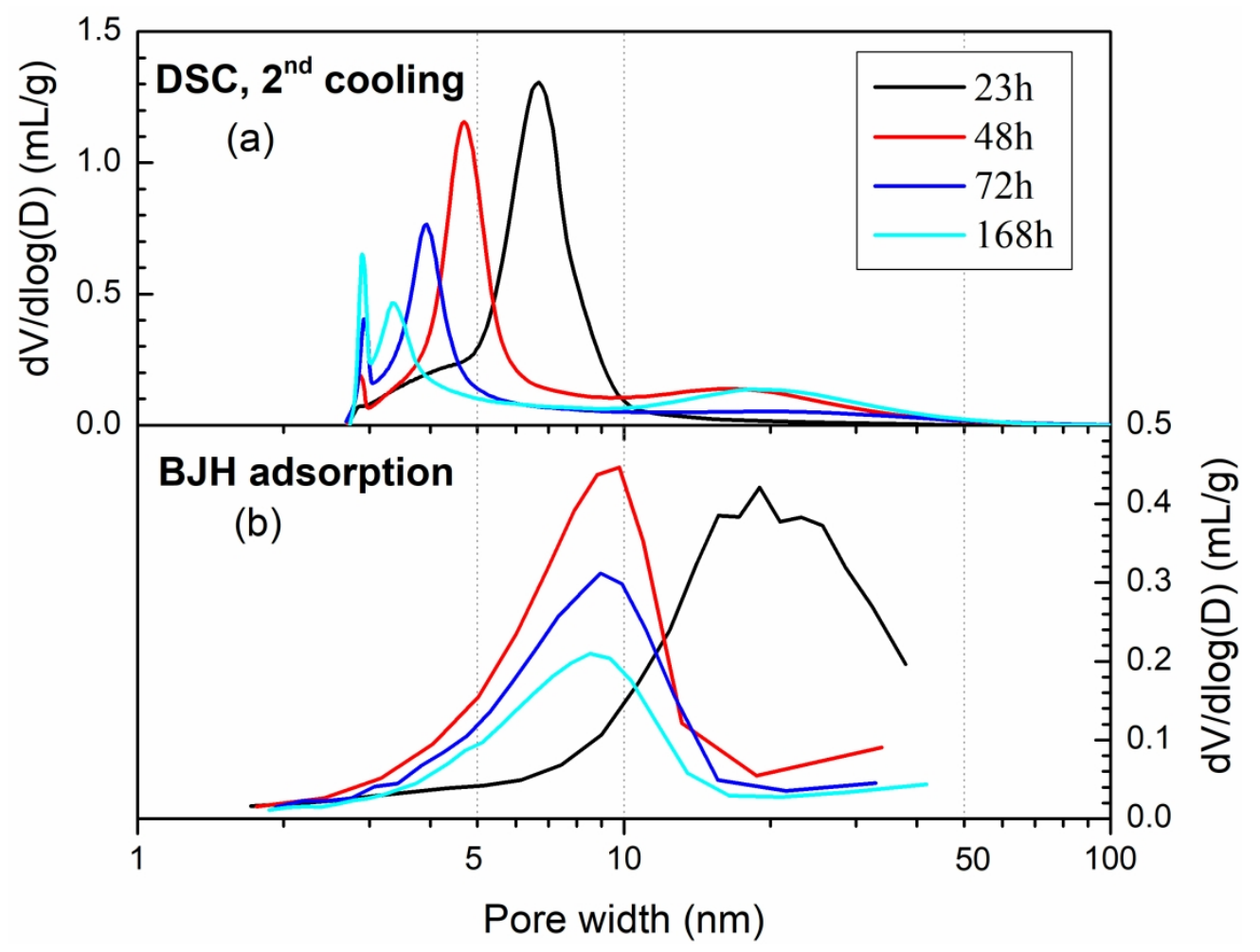

Figure 7 : Pore size distribution of washed sodium geopolymer obtained by thermoporometry (second cooling sequence) and nitrogen gas adsorption as a function of curing times. Note that the pore size range of application of the $\mathrm{BJH}$ analysis for nitrogen adsorption method $(\mathrm{BJH})$ is $2-50 \mathrm{~nm}$. In our case, the upper limit of thermoporometry is $325 \mathrm{~nm}$ since the cooling step starts at $-0.2{ }^{\circ} \mathrm{C}$.

$$
240 \times 183 \mathrm{~mm}(300 \times 300 \mathrm{DPI})
$$




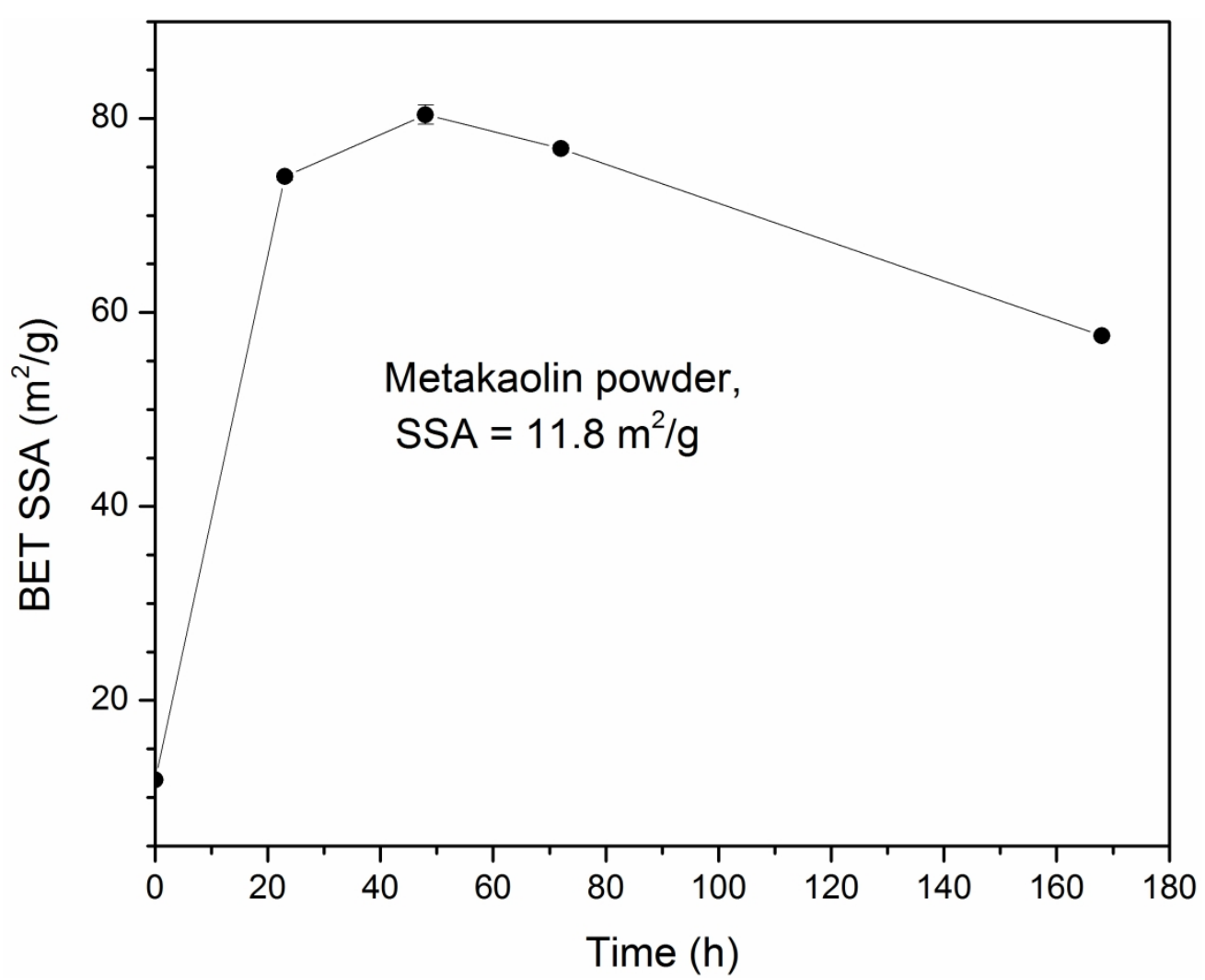

Figure 8 : Evolution of BET specific surface area obtained by nitrogen gas adsorption, as a function of curing times.

$225 \times 180 \mathrm{~mm}(300 \times 300 \mathrm{DPI})$ 


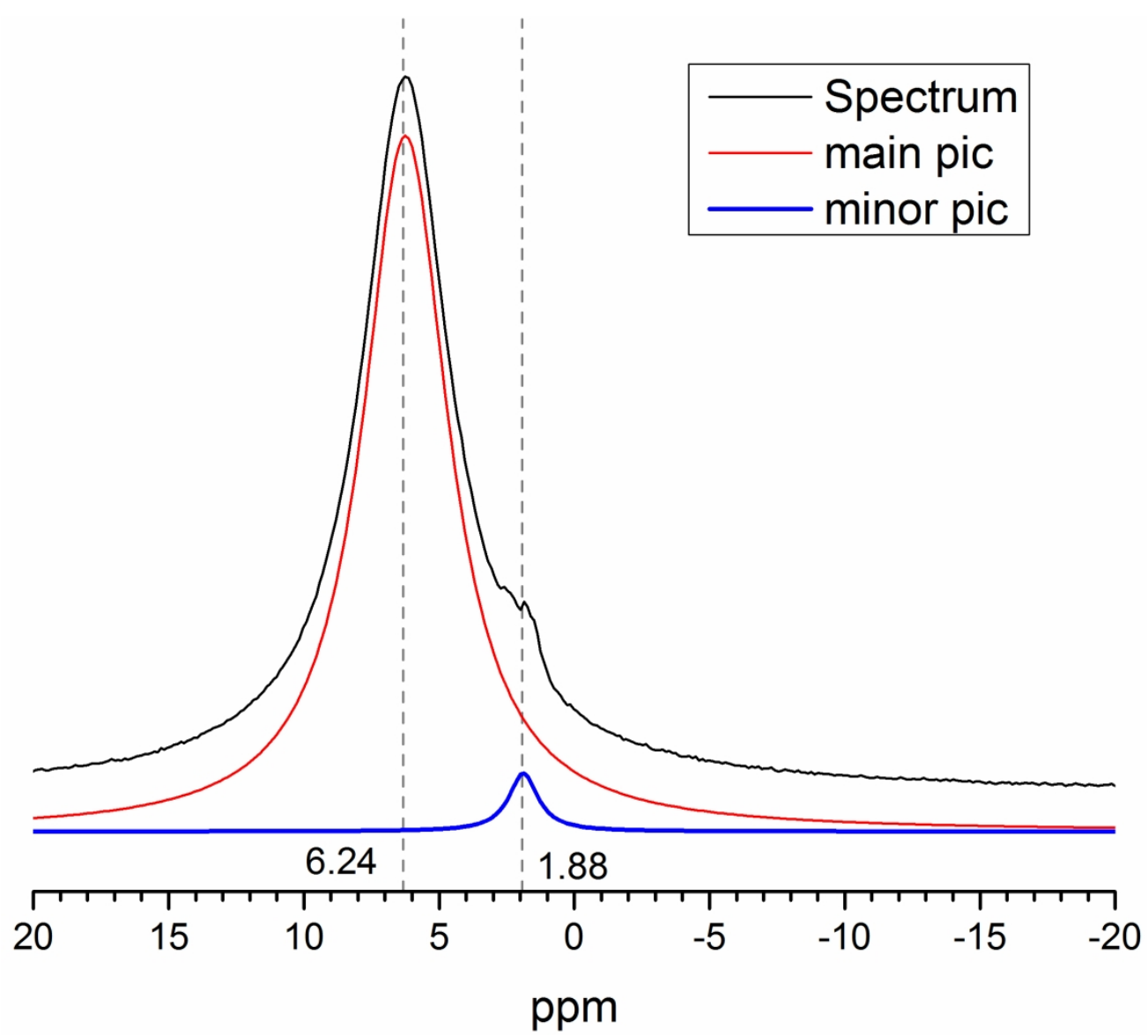

Figure $9: 1 \mathrm{H}$ NMR of $23 \mathrm{~h}$ lyophilized sodium geopolymer. The experimental spectrum (top trace) can be decomposed in two resonances, a minor one at $1.9 \mathrm{ppm}$ related to the protons of the silanol groups ( $\mathrm{SiOH})$, and a dominant one at $6.2 \mathrm{ppm}$ attributed to the protons in the sodium hydration water (lower traces).

$195 \times 173 \mathrm{~mm}(300 \times 300 \mathrm{DPI})$ 


\title{
SUPPLEMENTAL MATERIALS TO
}

\section{Evolution of the microstructure of unconsolidated geopolymers by thermoporometry}

\author{
Quang Hung Nguyen* a, Mohamed Hanafi ${ }^{a}$, Jan-Philip Merkl bł , Jean-Baptiste \\ d'Espinose de Lacaillerie * a
}

${ }^{a}$ Soft Matter Science and Engineering (SIMM), UMR CNRS 7615, PSL Research

University, ESPCI Paris, 75005, Paris, France

${ }^{b}$ BASF Construction Solutions GmbH, Dr.-Albert-Frank-Straße 32, 83308 Trostberg, Germany 


\section{Estimation of the sodium concentration of the pore solution after the washing steps.}

The following back-of-the-envelope calculations aim at checking if the pore solution after washing behaves as pure water despite the cation exchange capacity (CEC) of sodium geopolymers.

The geopolymer (GP) being washed with Milli-Q water, sodium cations can only exchange with hydronium ions according to:

$[\mathrm{GP}]^{-} \cdot \mathrm{Na}^{+}+\mathrm{H}_{3} \mathrm{O}^{+} \leftrightarrow[\mathrm{GP}]^{-} \cdot \mathrm{H}_{3} \mathrm{O}^{+}+\mathrm{Na}^{+}$

The CEC of geopolymers and the equilibrium constant of this exchange reaction is not known due to the amorphous nature of geopolymers and to their complex structures. We thus based our calculations on three hypothetical situations corresponding to different exchange ratios ( $r$ ): (1) a complete $100 \%$ exchange, (2) a $15 \%$ exchange, (3) a 1\% exchange. The complete exchange (1) is unrealistic. The $15 \%$ exchange (2) was selected by analogy with zeolites were a $15 \%$ overwashing is commonly observed ((Sherry, H., Howard, S., 2003. Ion exchange. Handbook of Zeolite Science and Technology, pages 1007-1061, Marcel Dekker, New York).

On each washing step, $5 \mathrm{~g}$ of geopolymer is equilibrated with $500 \mathrm{~mL}(V)$ of pure (Milli-Q) water. Considering that the pore volume fraction of the synthesized geopolymers was about $50 \%$ (see Table 3 in the main text) and its bulk density was about $1.1 \mathrm{~mL} \cdot \mathrm{g}^{-1}, 5 \mathrm{~g}$ of geopolymer corresponded to a pore solution volume $\left(V_{p}\right)$ of about $2.75 \mathrm{~mL}$.

Based on the nominal composition of the geopolymer synthesized here, 3. $6 \mathrm{SiO}_{2} \cdot \mathrm{Al}_{2} \mathrm{O}_{3} \cdot \mathrm{Na}_{2} \mathrm{O} \cdot 12 \mathrm{H}_{2} \mathrm{O}, 5 \mathrm{~g}$ of geopolymer corresponds to $0.52 \mathrm{~g}$ of $\mathrm{Na}_{2} \mathrm{O}$ and thus to a starting amount of sodium $\left(n_{N a}\right)$ of $17 \mathrm{mmol}$. After one washing step, assuming that at equilibrium the pore solution had the same composition as the one of the washing bath and neglecting variation of the density of water with sodium concentration, one estimates the final sodium cation concentration in the pores to be: 


$$
\left[N a^{+}\right] \cong \frac{r \times n_{N a}}{\left(V+V_{p}\right)}
$$

The normal freezing point depression $\left(\Delta T_{f p}\right)$ of $1 \mathrm{~kg}$ of bulk water by $\mathrm{NaOH}$ varies between 3.44 ${ }^{\circ} \mathrm{C}$ and $3.72{ }^{\circ} \mathrm{C}$ per mole of $\mathrm{NaOH}$, depending on authors and concentration ranges. Neglecting temperature effects and water contraction around the sodium cations, that is considering that $1 \mathrm{~L}$ of liquid corresponds to $1 \mathrm{~kg}$, the freezing point depression of the pore solution is given by $\Delta T \cong\left[N a^{+}\right] \times \Delta T_{b f}$

Putting numerical values into Eq.1 and Eq. 2, leads to final concentrations after one washing step of $0.03^{\circ} \mathrm{C}, 0.005^{\circ} \mathrm{C}$, and $0.0003^{\circ} \mathrm{C}$ for exchange ratios of $100 \%, 15 \%$ and $1 \%$ respectively. After two or three washing steps, those values can only decrease. In all cases, the expected sodium concentration in the pore solution at equilibrium after washing should thus lead to negligible (well below a hundreth of a ${ }^{\circ} \mathrm{C}$ ) freezing point depression. The pore solution could thus be safely considered to behave as pure water. 


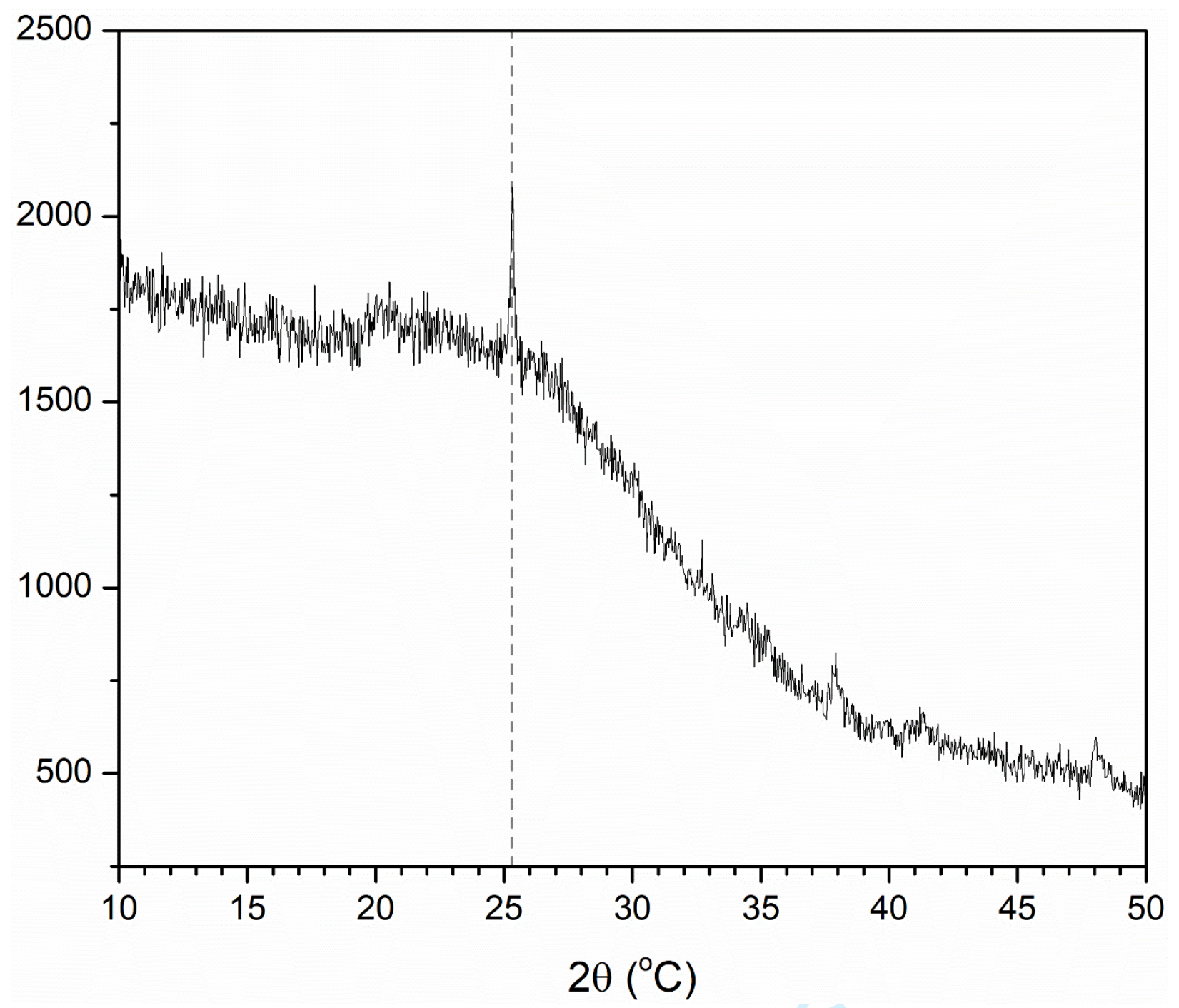

Fig S1: XRD of cured sodium geopolymer at $23 \mathrm{~h}$. The vertical dashed line indicates the position of XRD peak of anatase $\left(\mathrm{TiO}_{2}\right)$ 


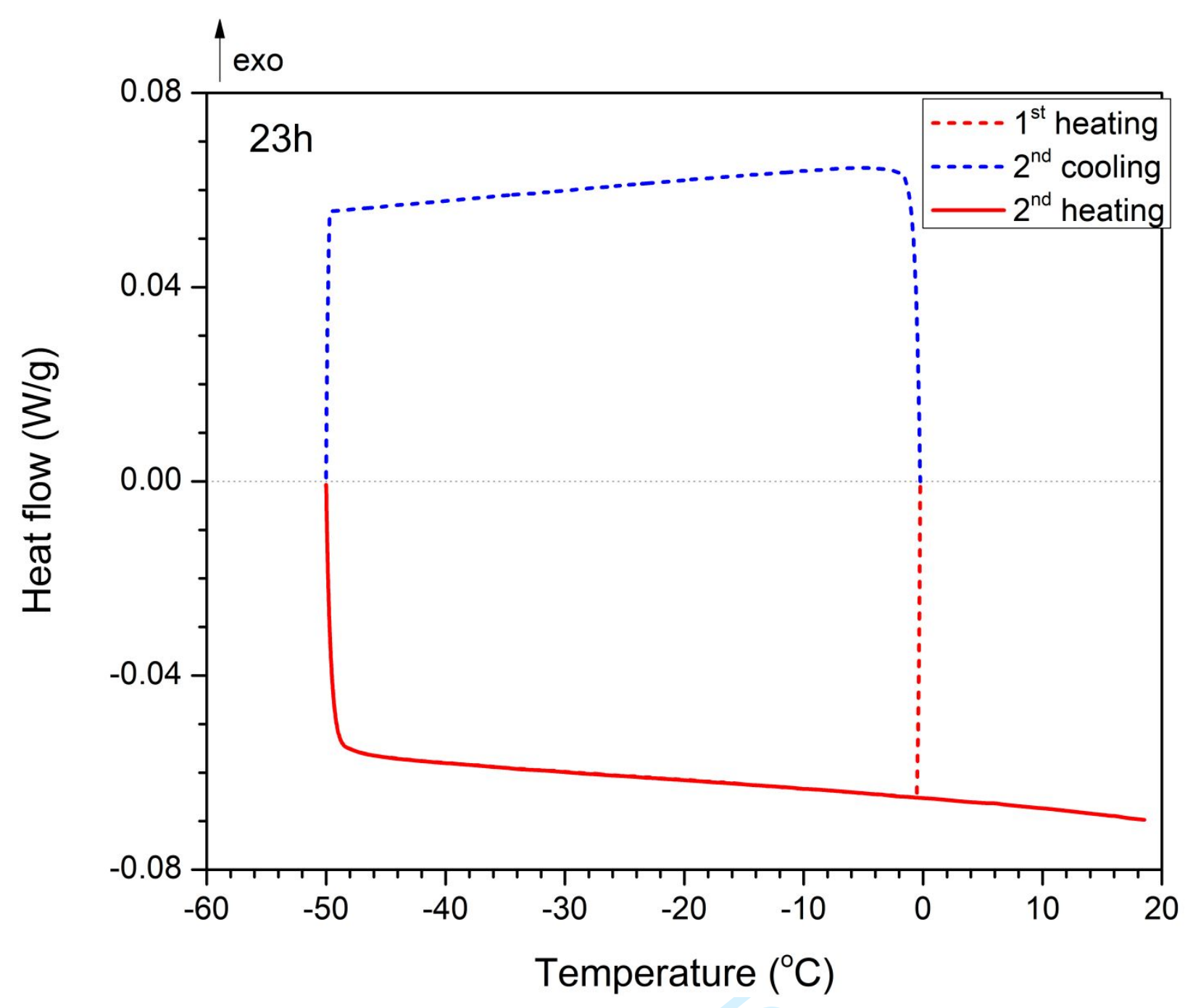

Figure S2: Thermogram of $23 \mathrm{~h}$ freeze-dried sodium geopolymer paste 


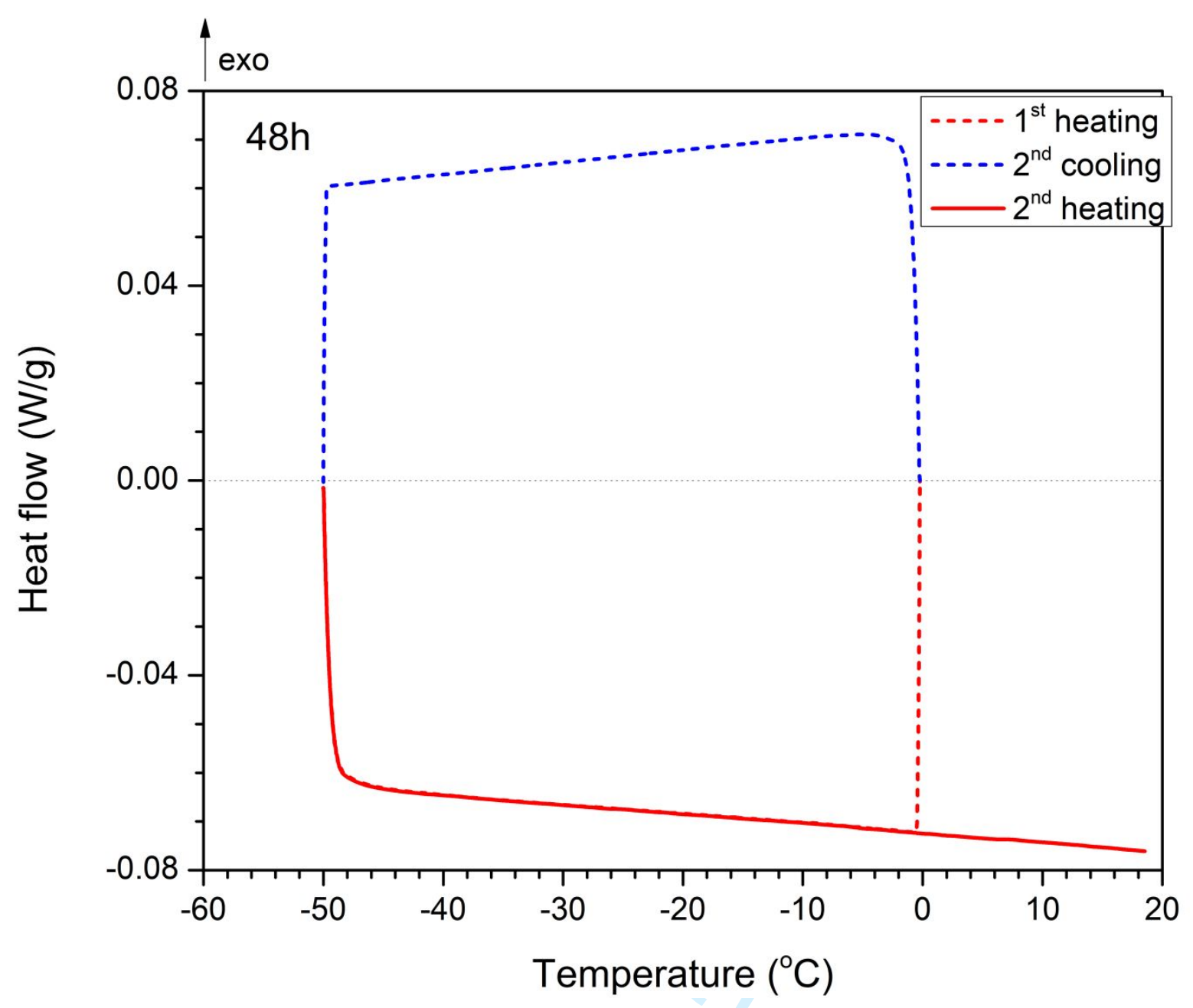

Figure S3: Thermogram of 48h freeze-dried washed sodium geopolymer paste. 


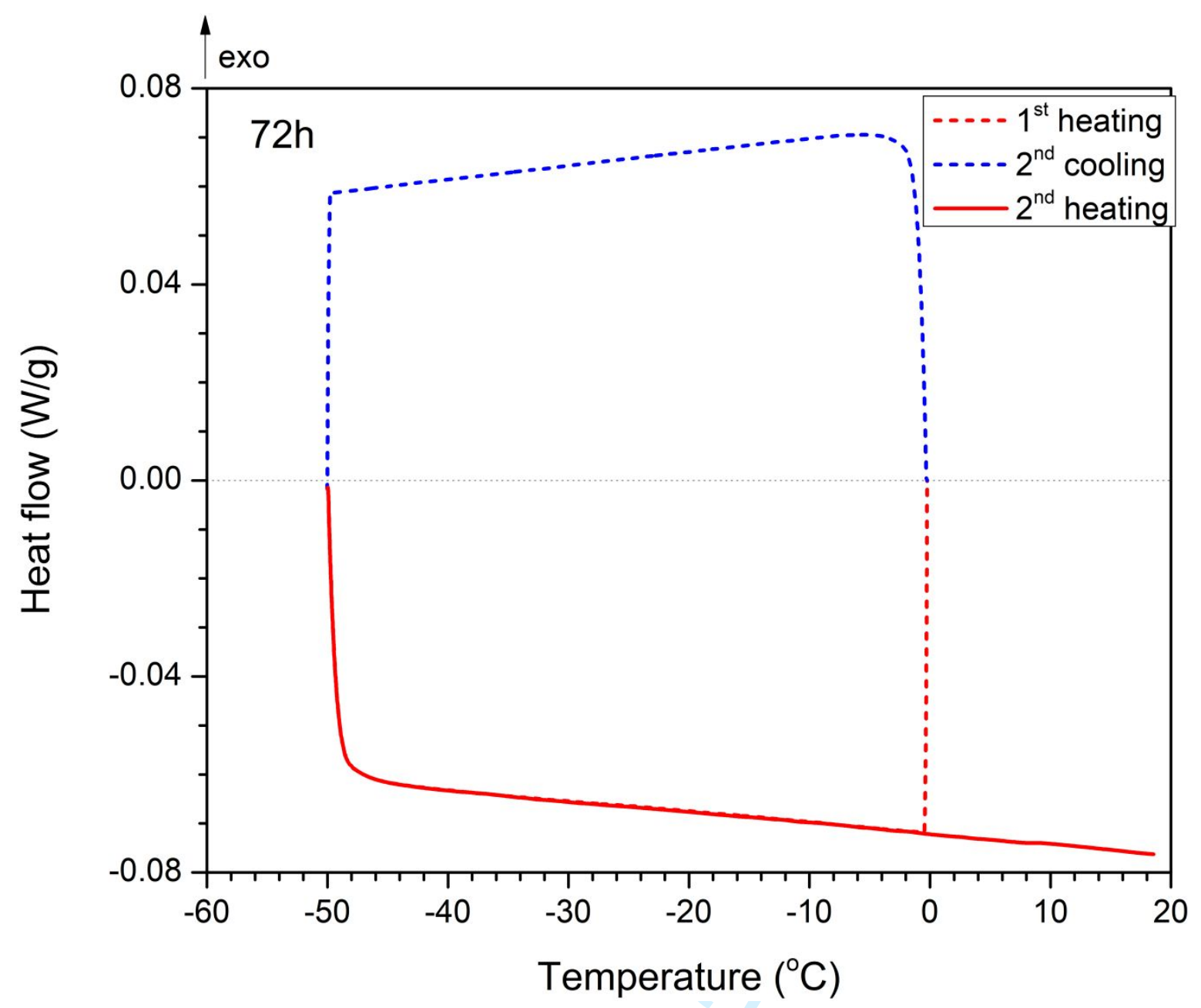

Figure S4: DSC thermogram of $72 \mathrm{~h}$ freeze-dried washed sodium geopolymer paste 


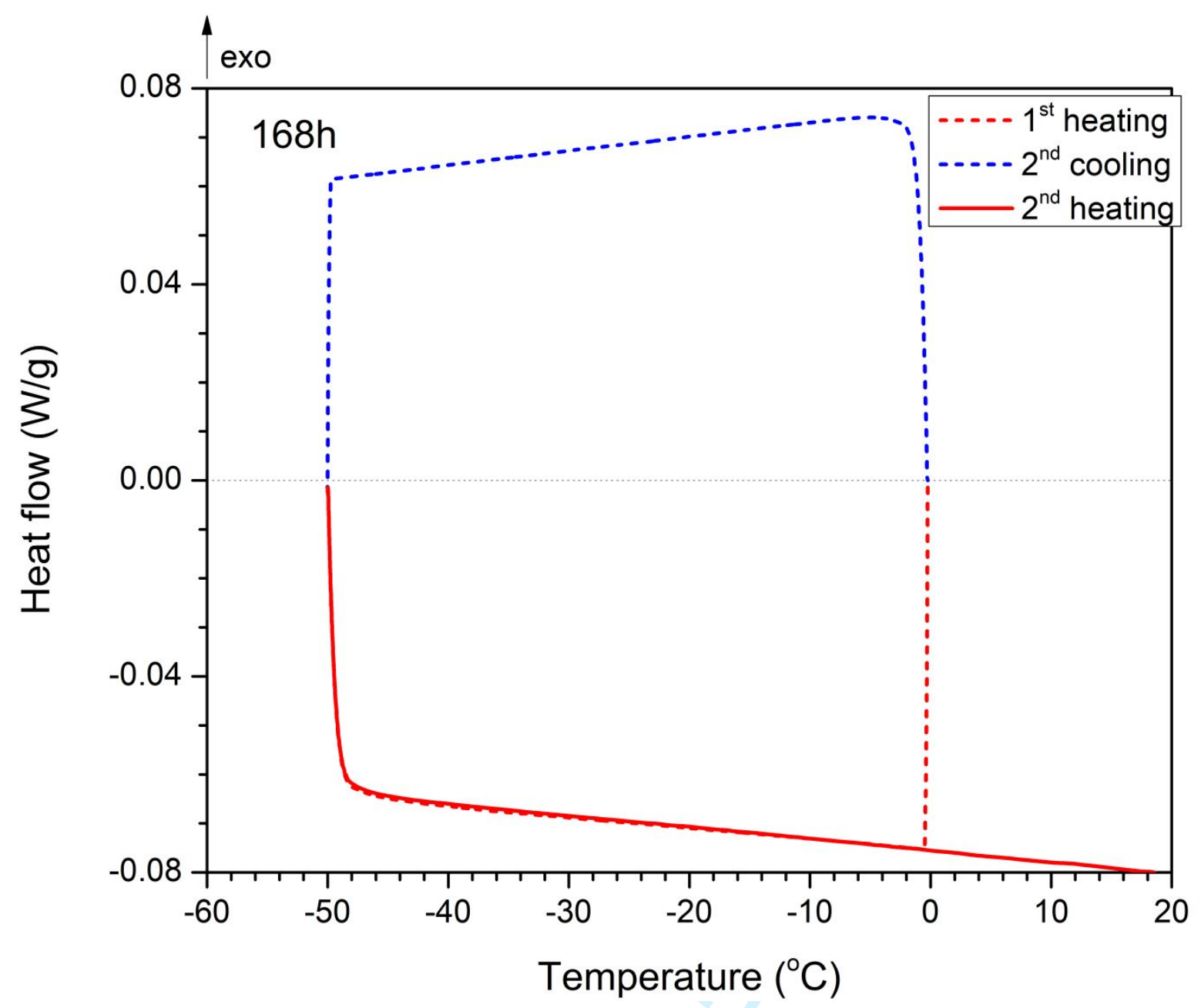

Figure S5: DSC thermogram of $168 \mathrm{~h}$ freeze-dried washed sodium geopolymer paste 

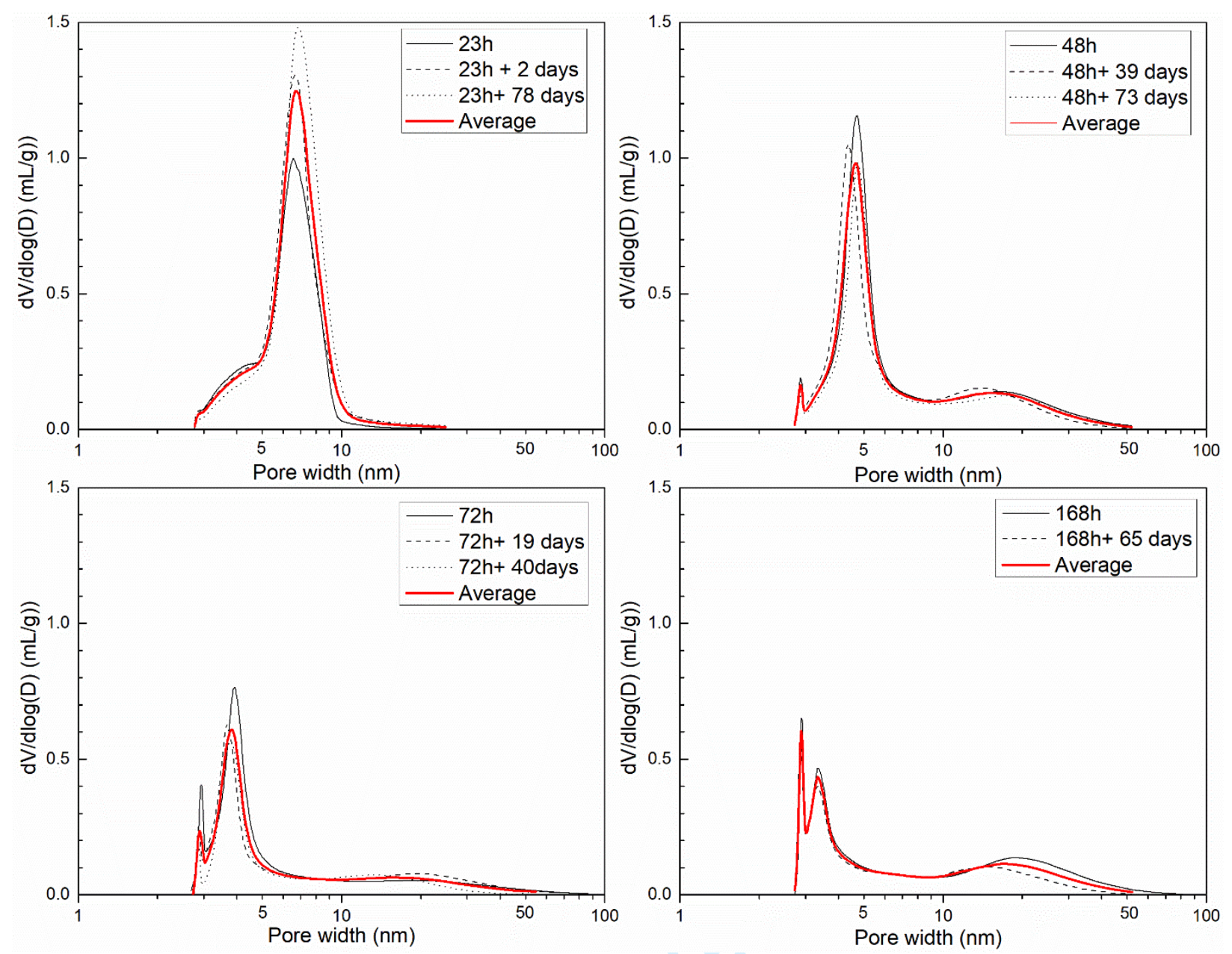

Figure S6: Pore size distribution of a washed sodium geopolymer, obtained by thermoporometry (second cooling sequence), as a function of curing times (23 h, $48 \mathrm{~h}, 72 \mathrm{~h}$ and $168 \mathrm{~h}$ ). The DSC was repeated at various time intervals after washing (solid lines: immediately after washing, dotted and dashed lines: at times indicated on the figure). From this experiments, a variation on estimated pore volumes due to experimental errors and aging was of about $10 \%$. The overall shape of the PSD was not changed though after stoppage. 